\title{
Asymptotic behavior of the exchange-correlation energy density and the Kohn-Sham potential in density functional theory: exact results and strategy for approximations
}

\author{
Eli Kraisler \\ Fritz Haber Center for Molecular Dynamics and Institute of Chemistry, \\ The Hebrew University of Jerusalem, 9091401 Jerusalem, Israel
}

(Dated: August 18, 2019)

\begin{abstract}
Density functional theory (DFT) is nowadays the leading theoretical framework for quantum description of materials from first principles. Being an exact theory on one hand and computationally efficient on the other hand, DFT allows to address large and complex many-electron systems and accurately predict their properties. The predictive power of DFT critically depends though on an accurate approximation to the generally unknown exchange-correlation (xc) energy functional. Approximations can be constructed from first principles by satisfying known properties of the exact functional. In this work I review two such exact properties: the asymptotic behavior of the xc energy density per particle and the asymptotic behavior of the Kohn-Sham potential, in finite many-electron systems. The derivation of the asymptotic forms for both quantities is reviewed, employing the concepts of the adiabatic connection and of the xc hole with relation to the first quantity and the electron exact factorization approach for the second one. Furthermore, it is shown that the correct asymptotic behavior of one of the aforementioned quantities does not guarantee a correct behavior of the other. In this process, a new quantity, the xc hole response function, is defined and its exact exchange part is analytically derived. The extent to which existing xc approximations satisfy the named exact properties is reviewed and the relationship between correct asymptotics and freedom from one-electron self-interaction in DFT is discussed. Finally, a strategy for development of advanced approximations for exchange and correlation with a correct asymptotic behavior is suggested.
\end{abstract}

\section{INTRODUCTION}

Computational modelling of materials and reliable prediction of their properties is a highly important scientific task with vast technological applications. Our ability to simulate materials, even before they are synthesized in the lab, has dramatically improved over the recent years. Growth in available computational resources, as well as development of dedicated software for materials simulations, allow scientists nowadays to address large, complex and heterogeneous systems (see, e.g., [1-7]). In many cases, accurate description of materials' properties requires modelling on the level of the electron-electron interactions, and therefore the system has to be addressed quantum-mechanically. Density functional theory (DFT) [8-20] became in recent decades the leading theoretical framework to provide such a description. Being an exact theory on one hand and allowing a computationally efficient implementation on the other hand, with a favourable scaling of the numerical effort with the system size, DFT makes it possible to address in practice large many-electron systems and to be able to accurately predict their properties.

DFT is built on solid theoretical foundations: back in 1964, Hohenberg and Kohn [21] have shown that the ground-state electron density, $n(\mathbf{r})$, includes essentially all the information needed to describe a given manyelectron system, be it a single atom, a molecule, a nano cluster, a crystalline solid or a DNA [197]. This is due to the one-to-one correspondence [198] between the ground state density $n(\mathbf{r})$ and $v_{\text {ext }}(\mathbf{r})$ - the external potential felt by the electrons, primarily due to their Coulomb attrac- tion to the nuclei. As a result, any physical quantity can be viewed as a functional of the the density $n(\mathbf{r})$, including the total energy, $E[n]$. Importantly, $E[n]$ is a variational quantity: by varying the density, for a given external potential $v_{\text {ext }}(\mathbf{r})$, one can find the minimum of the energy functional, which is the true ground-state energy of the system, and the corresponding minimizing density is the corresponding ground-state density [22, 23].

To obtain the density and the energy in practice, we usually follow the approach by Kohn and Sham (KS) [24]: we introduce a system of non-interacting electrons, which feel an effective (KS) potential, $v_{\mathrm{KS}}(\mathbf{r})$, chosen in such a way that the ground-state density of the non-interacting system equals exactly the ground-state density of the original, interacting system of interest. The KS potential is normally expressed as

$$
v_{\mathrm{KS}}[n](\mathbf{r})=v_{\mathrm{ext}}(\mathbf{r})+v_{\mathrm{H}}[n](\mathbf{r})+v_{\mathrm{xc}}[n](\mathbf{r}),
$$

being the sum of the external potential, $v_{\text {ext }}(\mathbf{r})$, the Hartree potential, $v_{\mathrm{H}}[n](\mathbf{r})=\int d^{3} r^{\prime} n\left(\mathbf{r}^{\prime}\right) /\left|\mathbf{r}-\mathbf{r}^{\prime}\right|[199]$, which corresponds to the classical electrostatic repulsion of the 'electron cloud', and the exchange-correlation (xc) potential, $v_{\mathrm{xc}}[n](\mathbf{r})$, which is responsible for all the quantum interactions between the electrons [200]. This last term is generally unknown and has to be approximated in any practical calculation.

Once the KS potential is given, we can actually treat the KS electrons independently due to the lack of interaction in the KS system, solve the one-electron Schrödinger equation,

$$
\left(-\frac{1}{2} \nabla^{2}+v_{\mathrm{KS}}[n](\mathbf{r})\right) \varphi_{i}(\mathbf{r})=\varepsilon_{i} \varphi_{i}(\mathbf{r}),
$$


and construct the density from the first $N$ low-lying levels: $n(\mathbf{r})=\sum_{i=1}^{N}\left|\varphi_{i}(\mathbf{r})\right|^{2}$. The ground state energy is then obtained as

$$
E[n]=T_{\mathrm{KS}}[n]+\int d^{3} r v_{\mathrm{ext}}(\mathbf{r}) n(\mathbf{r})+E_{\mathrm{H}}[n]+E_{\mathrm{xc}}[n],
$$

with $T_{\mathrm{KS}}[n]$ being the KS kinetic energy, $E_{\mathrm{H}}(\mathbf{r})=$ $\frac{1}{2} \iint d^{3} r d^{3} r^{\prime} n(\mathbf{r}) n\left(\mathbf{r}^{\prime}\right) /\left|\mathbf{r}-\mathbf{r}^{\prime}\right|$ being the Hartree (electrostatic) energy and $E_{\mathrm{xc}}[n]$ being the (again, unknown) $x c$ energy. The relation between the xc energy and the xc potential is that of a functional derivative: $v_{\mathrm{xc}}[n](\mathbf{r})=$ $\delta E_{\mathrm{xc}} / \delta n(\mathbf{r})$. The xc energy is often expressed as

$$
E_{\mathrm{xc}}[n]=\int d^{3} r n(\mathbf{r}) e_{\mathrm{xc}}[n](\mathbf{r}),
$$

where $e_{\mathrm{xc}}[n](\mathbf{r})$ is termed the exchange-correlation energy density per particle. It is $e_{\mathrm{xc}}[n](\mathbf{r})$ and $v_{\mathrm{xc}}[n](\mathbf{r})$ that we will focus on in this article.

Approximating the xc energy functional is the central approximation made in KS-DFT. It has attracted much scientific effort during the years [25-27], because the predictive power of the method crucially depends on the quality of the xc approximation used (see, e.g., [28-34]).

One strategy for development of accurate xc approximations from first principles is the strategy of satisfying constraints [35-37]: Although the exact xc functional is not known, many of its properties are. By constructing an xc approximation in a constrained manner, such that it satisfies known exact properties, we aim to increase its predictive power and widen its domain of applicability. The Perdew-Burke-Ernzerhof (PBE) generalized gradient approximation (GGA) [38] and the SCAN metaGGA $[39,40]$ are examples of successful implementation of this strategy.

Many exact properties of the xc functional have been revealed during the years [41-70]. The importance of discovering new properties of the exact xc functional and understanding the relationship between them $[71,72]$ can hardly be underestimated. In this work we focus on the asymptotic behavior of the xc potential, $v_{\mathrm{xc}}[n](\mathbf{r})$, and the xc energy density per particle, $e_{\mathrm{xc}}[n](\mathbf{r})$, in finite systems, namely on the properties of $v_{\mathrm{xc}}$ and $e_{\mathrm{xc}}$ in regions that are far from where the nuclei and (most of the) electrons of system are. Correct asymptotics of the aforementioned quantities is important in various physical and chemical scenarios: it helps to obtain stable anions [10], it is important for satisfying the ionization potential (IP) theorem in DFT [45, 46, 48, 50, 52, 73, 74], which connects the highest KS eigenvalue to the IP, it leads to unoccupied eigenvalues of improved interpretability, and as a consequence allows for higher accuracy in the prediction of various response properties [75-77]. Moreover, correct asymptotics proved to be key for developing such an important class of functionals as range-separated hybrids (RSHs) [2, 78-82]. Finally, correct asymptotic behavior of a given xc approximation is strongly associated with freedom from the spurious self-interaction [43] of electrons in KS-DFT, an association which has been recently reviewed in Ref. [71] and is further discussed below. In this work we confine ourselves to systems with a constant and integer number of electrons, $N$ (but see [72, 83-86] and references therein for systems with fractional $N$ ). Furthermore, we choose to treat systems whose highest occupied KS orbital does not possess nodal planes (see [71, 87-93] for the latter case).

The rest of the article is organized as follows. In Sec. II the derivation of the the asymptotic behavior of the exact $e_{\mathrm{xc}}[n](\mathbf{r})$ and $v_{\mathrm{xc}}[n](\mathbf{r})$ is reviewed. Then in Sec. III it is analytically shown that the correct asymptotic behavior of $e_{\mathrm{xc}}[n](\mathbf{r})$ does not guarantee a correct behavior of $v_{\mathrm{xc}}[n](\mathbf{r})$ and vice versa. This happens because the asymptotic forms of these two quantities are related to two different properties of the xc hole (to be defined below): the sum rule of the hole and the behavior of the xc hole response function - a new quantity, which we define and explore below. In Sec. IV we analytically derive the exact exchange part of the aforementioned response function employing the optimized effective potential (OEP) method. In Sec. V we discuss how well the the exact asymptotic behavior of $v_{\mathrm{xc}}$ and $e_{\mathrm{xc}}$ is reproduced in various xc approximations and in Sec. VI we discuss the relationship between correct asymptotics and freedom from one-electron self-interaction. Finally, in Sec. VII we discuss how the findings presented in this work can be used to formulate a strategy for development of advanced approximations for exchange and correlation with a correct asymptotic behavior.

\section{FORMALISM}

\section{A. Exchange-correlation energy-density per particle}

The exchange-correlation energy-density per particle, $e_{\mathrm{xc}}[n](\mathbf{r})$, is usually introduced by Eq. (4), which essentially means that the infinitesimal contribution to the xc energy from the infinitesimal volume element $d^{3} r$ around $\mathbf{r}$ equals $n(\mathbf{r}) \cdot e_{\mathrm{xc}}[n](\mathbf{r}) d^{3} r$. Normalized by the number of electrons in this volume, $n(\mathbf{r}) d^{3} r$, the xc energy-density per particle is therefore $e_{\mathrm{xc}}[n](\mathbf{r})$.

Equation (4) does not define $e_{\mathrm{xc}}[n](\mathbf{r})$ uniquely, but only up to any additive function $f(\mathbf{r})$, which obeys $\int d^{3} r n(\mathbf{r}) f(\mathbf{r})=0$ (see, e.g., Refs. [64, 94-96] for further details) [201]. However, to discuss the asymptotics at $|\mathbf{r}| \rightarrow \infty$ we must define $e_{\mathrm{xc}}[n](\mathbf{r})$ unambiguously. This can be done using the concepts of the xc hole and of the adiabatic connection. In the following, we describe these concepts in some detail.

First, we introduce the concept of the xc hole, following Ref. [10] (see also [9, ch. 8.5], [19, 43, 97-99]). We start from the two-electron reduced density matrix, $\rho_{2}\left(\mathbf{r}, \mathbf{r}^{\prime}\right)$, which is the probability density to find an electron at $\mathbf{r}$ and another electron at $\mathbf{r}^{\prime}$. In terms of the many-electron 
wavefunction $\Psi\left(\mathbf{r}_{1} \sigma_{1}, \mathbf{r}_{2} \sigma_{2}, \mathbf{r}_{3} \sigma_{3}, \cdots, \mathbf{r}_{N} \sigma_{N}\right)$,

$$
\begin{array}{r}
\rho_{2}\left(\mathbf{r}, \mathbf{r}^{\prime}\right)=N(N-1) \int d^{3} r_{3} \cdots d^{3} r_{N} \sum_{\sigma_{1}} \cdots \sum_{\sigma_{N}} \\
\left|\Psi\left(\mathbf{r} \sigma_{1}, \mathbf{r}^{\prime} \sigma_{2}, \mathbf{r}_{3} \sigma_{3}, \cdots, \mathbf{r}_{N} \sigma_{N}\right)\right|^{2}
\end{array}
$$

Note that $\rho_{2}\left(\mathbf{r}, \mathbf{r}^{\prime}\right)$ is symmetric in $\mathbf{r}$ and $\mathbf{r}^{\prime}$. Next, we define $n_{2}\left(\mathbf{r}^{\prime} \mid \mathbf{r}\right)$ - the conditional probability density of finding an electron at $\mathbf{r}^{\prime}$, given there is an electron at $\mathbf{r}$. It is related to $\rho_{2}\left(\mathbf{r}, \mathbf{r}^{\prime}\right)$ as follows: $\rho_{2}\left(\mathbf{r}, \mathbf{r}^{\prime}\right)=n(\mathbf{r}) n_{2}\left(\mathbf{r}^{\prime} \mid \mathbf{r}\right)$. Unlike $\rho_{2}\left(\mathbf{r}, \mathbf{r}^{\prime}\right)$, the conditional density $n_{2}\left(\mathbf{r}^{\prime} \mid \mathbf{r}\right)$ is not symmetric in $\mathbf{r}$ and $\mathbf{r}^{\prime} . n_{2}\left(\mathbf{r}^{\prime} \mid \mathbf{r}\right)$ can be presented as

$$
n_{2}\left(\mathbf{r}^{\prime} \mid \mathbf{r}\right)=n\left(\mathbf{r}^{\prime}\right)+n_{\mathrm{xc}}\left(\mathbf{r}^{\prime} \mid \mathbf{r}\right)
$$

where $n_{\mathrm{xc}}\left(\mathbf{r}^{\prime} \mid \mathbf{r}\right)$ is termed the xc hole. It can be thought of as the reduction in the electron density (creation of a 'hole') at some point $\mathbf{r}^{\prime}$ because there exists an electron at $\mathbf{r}$. From the simple observation that $\int d^{3} r^{\prime} \rho_{2}\left(\mathbf{r}, \mathbf{r}^{\prime}\right)=$ $(N-1) n(\mathbf{r})$ follows the so-called sum rule for the xc hole:

$$
\int d^{3} r^{\prime} n_{\mathrm{xc}}\left(\mathbf{r}^{\prime} \mid \mathbf{r}\right)=-1
$$

This is an analytical result of high importance, which will serve us below.

Now we introduce the concept of the adiabatic connection [90, 97, 99-101]. We consider a series of manyelectron systems with a varying strength of the electronelectron repulsion. Formally, these systems are described by the the Hamiltonian

$$
\hat{H}^{(\lambda)}=\hat{T}+\hat{V}^{(\lambda)}+\lambda \hat{W}
$$

where $\hat{T}=-\frac{1}{2} \sum_{i} \nabla_{i}^{2}$ is the kinetic energy operator, $\hat{V}=\sum_{i} v_{\text {ext }}^{(\lambda)}\left(\mathbf{r}_{i}\right)$ is the potential energy operator (its $\lambda$-dependence is clarified below) and $\hat{W}=$ $\frac{1}{2} \sum_{i} \sum_{j \neq i}\left|\mathbf{r}_{i}-\mathbf{r}_{j}\right|^{-1}$ is the usual electron-electron repulsion operator, which is preceded in the Hamiltonian by the parameter $\lambda$. This parameter defines the strength of the electron-electron repulsion; we consider the range of values $0 \leqslant \lambda \leqslant 1$. Next, we introduce the following restriction: the ground state density of all these Hamiltonians is the same, $n(\mathbf{r})$. The external potential $v_{\text {ext }}^{(\lambda)}(\mathbf{r})$ is chosen to satisfy this restriction, and therefore it is different for each $\lambda$. As a result, the ground state energy of the system can be written as

$$
E^{(\lambda)}=\left\langle\Psi^{(\lambda)}\left|\hat{H}^{(\lambda)}\right| \Psi^{(\lambda)}\right\rangle=\int d^{3} r v_{\text {ext }}^{(\lambda)}(\mathbf{r}) n(\mathbf{r})+F^{(\lambda)}[n],
$$

where $\left|\Psi^{(\lambda)}\right\rangle$ is the ground state of the $\lambda$-system and $F^{(\lambda)}[n]:=\left\langle\Psi^{(\lambda)}|\hat{T}+\lambda \hat{W}| \Psi^{(\lambda)}\right\rangle$. The particular case of $\lambda=1$ yields the fully-interacting many-electron system, where $v_{\text {ext }}^{(1)}(\mathbf{r})$ equals the external potential $v_{\text {ext }}(\mathbf{r}), E^{(1)}$ equals the total energy $E[n]$ of Eq. $(3), F^{(1)}[n]$ is termed the Hohenberg-Kohn functional $[8,21]$ and $\left|\Psi^{(1)}\right\rangle$ is the fully-interacting many-electron ground state. The case of $\lambda=0$ yields the KS system, where $v_{\text {ext }}^{(0)}(\mathbf{r})=v_{\mathrm{KS}}(\mathbf{r})$, $F^{(0)}[n]=T_{\mathrm{KS}}[n]$ and $\left|\Psi^{(0)}\right\rangle$ is the $\mathrm{KS}$ ground state denoted $\left|\Phi_{\mathrm{KS}}\right\rangle$, which, in the case of a pure ground state, is represented by a Slater determinant of the orbitals $\varphi_{i}(\mathbf{r})$. Comparing Eqs. (9) and (3), we can express the Hartree-exchange-correlation (Hxc) energy as $E_{\mathrm{H}}[n]+$ $E_{\mathrm{xc}}[n]=F^{(1)}[n]-F^{(0)}[n]$. Realizing that the righthand side can be written as $\int_{0}^{1} d \lambda d F^{(\lambda)}[n] / d \lambda$ and utilizing the Hellmann-Feynman theorem (see, e.g., [102], [103, $\S 11],[10$, ch. 1.2.4]), we represent the Hxc energy as

$$
E_{\mathrm{H}}[n]+E_{\mathrm{xc}}[n]=\int_{0}^{1} d \lambda\left\langle\Psi^{(\lambda)}|\hat{W}| \Psi^{(\lambda)}\right\rangle .
$$

This is an interesting and important result: formally, the Hxc energy can be expressed as the $\lambda$-average of the expectation value of the electron-electron repulsion operator, $\hat{W}$.

Connecting the two concepts we introduced above, we first note that the expectation value of $\hat{W}$ can be expressed in terms of the $\lambda$-dependent two-electron reduced density matrix, $\rho_{2}^{(\lambda)}\left(\mathbf{r}, \mathbf{r}^{\prime}\right)[202]:\left\langle\Psi^{(\lambda)}|\hat{W}| \Psi^{(\lambda)}\right\rangle=$ $\frac{1}{2} \iint d^{3} r d^{3} r^{\prime} \rho_{2}^{(\lambda)}\left(\mathbf{r}, \mathbf{r}^{\prime}\right) /\left|\mathbf{r}-\mathbf{r}^{\prime}\right|$. Expressing $\rho_{2}^{(\lambda)}\left(\mathbf{r}, \mathbf{r}^{\prime}\right)$ in terms of the (now also $\lambda$-dependent) xc hole, $n_{\mathrm{xc}}^{(\lambda)}\left(\mathbf{r}^{\prime} \mid \mathbf{r}\right)$ (cf. Eq. 6), we find that the xc energy equals

$$
E_{\mathrm{xc}}[n]=\frac{1}{2} \iint d^{3} r d^{3} r^{\prime} \frac{n(\mathbf{r}) \bar{n}_{\mathrm{xc}}\left(\mathbf{r}^{\prime} \mid \mathbf{r}\right)}{\left|\mathbf{r}-\mathbf{r}^{\prime}\right|},
$$

where $\bar{n}_{\mathrm{xc}}\left(\mathbf{r}^{\prime} \mid \mathbf{r}\right)=\int_{0}^{1} d \lambda n_{\mathrm{xc}}^{(\lambda)}\left(\mathbf{r}^{\prime} \mid \mathbf{r}\right)$ is the $\lambda$-averaged xc hole. From Eq. (11) we see that the xc energy can be viewed as the electrostatic interaction between the ground state density and the $\lambda$-averaged xc hole.

Finally, from Eq. (11) it is possible to uniquely define (not deduce) the xc energy-density per particle in terms of the xc hole:

$$
e_{\mathrm{xc}}[n](\mathbf{r})=\frac{1}{2} \int d^{3} r^{\prime} \frac{\bar{n}_{\mathrm{xc}}\left(\mathbf{r}^{\prime} \mid \mathbf{r}\right)}{\left|\mathbf{r}-\mathbf{r}^{\prime}\right|} .
$$

Naturally, $e_{\mathrm{xc}}[n](\mathbf{r})$ of Eq. (12) and $E_{\mathrm{xc}}[n]$ of Eqs. (11) satisfy relation (4). Equation (12) is known as the conventional gauge for $e_{\mathrm{xc}}[n](\mathbf{r})[64]$.

Once $e_{\mathrm{xc}}[n](\mathbf{r})$ is defined, we can discuss its behavior in the limit $|\mathbf{r}| \rightarrow \infty$. In this limit, $\left|\mathbf{r}-\mathbf{r}^{\prime}\right| \rightarrow|\mathbf{r}|$, and therefore exploiting the sum rule for the xc hole (Eq. (7)), we realize that

$$
e_{\mathrm{xc}}[n](\mathbf{r}) \rightarrow-\frac{1}{2|\mathbf{r}|}
$$

Therefore, the asymptotic behavior of $e_{\mathrm{xc}}[n](\mathbf{r})$ is a direct result of the sum rule for the xc hole [203].

\section{B. Exchange-correlation potential}

In the following, we analytically derive the asymptotic form of the xc potential, $v_{\mathrm{xc}}[n](\mathbf{r})$. But first, to get an intuition about this asymptotic behavior, it is instructive 
to look at the KS potential, $v_{\mathrm{KS}}[n](\mathbf{r})$, as a whole. At $r \rightarrow \infty$ (here and below $r=|\mathbf{r}|$, and we use both notations interchangeably), the KS potential can be roughly viewed as the potential that is felt by an electron when it appears very far from the system (i.e., from the nuclei and the other electrons). Then, such an electron should feel the overall attraction of the nuclei, screened by all the other electrons of the system. Denoting the sum of all the nuclear charges by $Z$ and the total number of electrons in the system by $N$, we realize that $v_{\mathrm{KS}}[n](\mathbf{r}) \sim-(Z-(N-1)) / r$. [This is because the number of the other electrons is $N-1$.] The asymptotic behavior of the external potential is $v_{\text {ext }}(\mathbf{r}) \sim-Z / r$. The asymptotic behavior of the Hartree potential can be derived directly from its definition, taking the limit $\left|\mathbf{r}-\mathbf{r}^{\prime}\right| \rightarrow|\mathbf{r}|$; it is $v_{\mathrm{H}}[n](\mathbf{r}) \sim N / r$. Therefore, we conclude that according to our intuitive explanation the $\mathrm{xc}$ potential should behave as $v_{\mathrm{xc}}[n](\mathbf{r}) \sim-1 / r$.

The asymptotic behavior of the exact $v_{\mathrm{xc}}[n](\mathbf{r})$ has been analytically obtained in the literature, using several approaches (see [42, 48, 50, 91, 93] and references therein). In the following we obtain this result using the exact electron factorization (EEF) approach [104-107] to the many-electron problem. Notably, although the intuitive explanation given above is by no means a rigorous derivation, its essential point is a correct one: to analyze the asymptotic behavior of $v_{\mathrm{xc}}$, we will address an $N$-electron system in the process of ionization. This is common to all the approaches on the matter.

With the EEF approach, we first consider a fullyinteracting $N$-electron system, which is defined by the Hamiltonian $\hat{H}_{N}$ (as in Eq. (8), with $\lambda=1$ there) and is completely described by the $N$-electron ground-state wavefunction $\Psi_{N}\left(\mathbf{r}_{1} \cdots \mathbf{r}_{N}\right)$. The subscript $N$ emphasizes the number of electrons in the system. Next, without loss of generality [104], we express the wavefunction as $\Psi_{N}\left(\mathbf{r}_{1} \cdots \mathbf{r}_{N}\right)=\chi\left(\mathbf{r}_{N}\right) \phi\left(\mathbf{r}_{1} \cdots \mathbf{r}_{N-1} \mid \mathbf{r}_{N}\right)$. Here $\left|\chi\left(\mathbf{r}_{N}\right)\right|^{2}$ has the meaning of the marginal probability density, namely the probability to find an electron at $\mathbf{r}_{N}$, whereas $\left|\phi\left(\mathbf{r}_{1} \cdots \mathbf{r}_{N-1} \mid \mathbf{r}_{N}\right)\right|^{2}$ has the meaning of the conditional probability density, namely the probability to find an electron at $\mathbf{r}_{1}$ and an electron at $\mathbf{r}_{2}$, and so on, given there is an electron at $\mathbf{r}_{N}$. In fact, $\left|\chi\left(\mathbf{r}_{N}\right)\right|^{2}$ is nothing else but the normalized ground-state density, $n\left(\mathbf{r}_{N}\right) / N$.

In the following we denote integration over all variables, $\mathbf{r}_{1} \cdots \mathbf{r}_{N}$, by double Dirac brackets, $\langle\langle\ldots\rangle$, and integration over $\mathbf{r}_{1} \cdots \mathbf{r}_{N-1}$ only by single Dirac brackets, $\langle\ldots\rangle$. Thus, $\left\langle\left\langle\Psi_{N} \mid \Psi_{N}\right\rangle\right\rangle=\left(\int d^{3} r_{N} n\left(\mathbf{r}_{N}\right) / N\right) \cdot\langle\phi \mid \phi\rangle$, and therefore $\langle\phi \mid \phi\rangle=1$, for any value of $\mathbf{r}_{N}$. In other words, both $\chi$ and $\phi$ are normalized to 1 . Furthermore, $\left\langle\phi \mid \Psi_{N}\right\rangle=\chi\left(\mathbf{r}_{N}\right)$ and $\left\langle\phi\left|\hat{H}_{N}\right| \Psi_{N}\right\rangle=E_{N} \chi\left(\mathbf{r}_{N}\right)$, where $E_{N}$ is the ground-state energy of the $N$-electron system [204]. Now, let us examine closely the $N$-electron Hamiltonian, $\hat{H}_{N}$. It can be expressed as $\hat{H}_{N}=\hat{H}_{N-1}-\frac{1}{2} \nabla_{N}^{2}+$ $v_{\text {ext }}\left(\mathbf{r}_{N}\right)+\sum_{j=1}^{N-1}\left|\mathbf{r}_{j}-\mathbf{r}_{N}\right|^{-1}$. Using this expression, we can derive $\left\langle\phi\left|\hat{H}_{N}-E_{N-1}\right| \Psi_{N}\right\rangle$, which on one hand equals $-I \chi\left(\mathbf{r}_{N}\right)$ (where $I=E_{N-1}-E_{N}$ is the ionization po- tential (IP) of the system), and on the other hand equals $-\frac{1}{2} \nabla_{N}^{2} \chi\left(\mathbf{r}_{N}\right)+v_{\mathrm{KSP}}\left(\mathbf{r}_{N}\right) \chi\left(\mathbf{r}_{N}\right)$, where $[48,104]$

$$
\begin{aligned}
v_{\mathrm{KSP}}\left(\mathbf{r}_{N}\right)= & v_{\mathrm{ext}}\left(\mathbf{r}_{N}\right)+\left\langle\phi\left|\hat{H}_{N-1}-E_{N-1}\right| \phi\right\rangle+ \\
& +\int d^{3} r \frac{n_{\phi}\left(\mathbf{r} \mid \mathbf{r}_{N}\right)}{\left|\mathbf{r}-\mathbf{r}_{N}\right|}+\frac{1}{2}\left\langle\nabla_{N} \phi \mid \nabla_{N} \phi\right\rangle
\end{aligned}
$$

is termed the Kohn-Sham-Pauli potential [48, 108, 109]. In the latter equation $n_{\phi}\left(\mathbf{r} \mid \mathbf{r}_{N}\right)$ is the 'density' that corresponds to $\phi$, namely, $n_{\phi}\left(\mathbf{r} \mid \mathbf{r}_{N}\right)=(N-$ 1) $\int d^{3} r_{2} \cdots \int d^{3} r_{N-1}\left|\phi\left(\mathbf{r}, \mathbf{r}_{2} \cdots \mathbf{r}_{N-1} \mid \mathbf{r}_{N}\right)\right|^{2}$. To derive Eq. (14), we considered the sum of $\left\langle\phi\left|\hat{H}_{N}-E_{N-1}\right| \Psi_{N}\right\rangle$ and of its complex conjugate, $\left\langle\Psi_{N}\left|\hat{H}_{N}-E_{N-1}\right| \phi\right\rangle$, and took advantage of the fact that $\nabla_{N}\langle\phi \mid \phi\rangle$ and $\nabla_{N}^{2}\langle\phi \mid \phi\rangle$ are zero, due to the normalization of $\phi$. To summarize, the marginal probability density amplitude, $\chi\left(\mathbf{r}_{N}\right)$, satisfies a Schrödinger-like equation

$$
\left(-\frac{1}{2} \nabla_{N}^{2}+v_{\mathrm{KSP}}\left(\mathbf{r}_{N}\right)\right) \chi\left(\mathbf{r}_{N}\right)=-I \chi\left(\mathbf{r}_{N}\right)
$$

where the eigenvalue equals the (negative of the) IP of the system and the potential is formally given by Eq. (14). This equation is central in the orbital-free (OF) approach [110-116], which is an alternative to the KS approach in DFT (cf. Eq. (2)).

We now explore the particular case where $r_{N}$ is very large, i.e., the $N^{\text {th }}$ electron is far away from all the electrons and nuclei in the system. This is nothing else but the process of (a vertical) ionization. In this process, the parametric dependence of $\phi\left(\mathbf{r}_{1} \cdots \mathbf{r}_{N-1} \mid \mathbf{r}_{N}\right)$ on $\mathbf{r}_{N}$ disappears: indeed, the probability to find the $N-1$ electrons at certain positions $\mathbf{r}_{1} \cdots \mathbf{r}_{N-1}$ does not depend on some infinitely distant electron at $\mathbf{r}_{N}$. Therefore, for $r_{N} \rightarrow \infty$, $\phi$ becomes: (i) independent of $\mathbf{r}_{N}$; (ii) the ground state of the $(N-1)$-electron Hamiltonian, $\hat{H}_{N-1}$. Consequently, the fourth term of Eq. (14) vanishes due to (i), the second term there vanishes due to (ii) and in the third term $n_{\phi}$ becomes the ground-state density of $\hat{H}_{N-1}$. At the limit $\left|\mathbf{r}-\mathbf{r}_{N}\right| \rightarrow\left|\mathbf{r}_{N}\right|$, this third term yields $(N-1) / r_{N}$, and the overall KSP potential decays as $\sim-(Z-(N-1)) / r_{N}$.

The asymptotic form of the KSP potential allows us to find the asymptotic behavior of $\chi(\mathbf{r})$. Assuming that the asymptotic decay of $n(\mathbf{r})$, and therefore of $\chi(\mathbf{r})$, is isotropic (and therefore excluding the aforementioned case of nodal planes $[71,87,88,90-93])$, the Laplacian can be expressed as $\nabla^{2}=\frac{\partial^{2}}{\partial r^{2}}+\frac{2}{r} \frac{\partial}{\partial r}$. Substitution of this form for the Laplacian and of the decay of the KSP potential obtained earlier into Eq. (15) leads to an ordinary differential equation for $\chi(r)$, whose solution is asymptotically given by $\chi(r) \sim r^{\beta} e^{-\sqrt{2 I} r}$, with $\beta=-1+(Z-N+1) / \sqrt{2 I}[42,93]$.

Next, from the perspective of the KS system, the density is the sum of the squares of all occupied KS orbitals, $\left|\varphi_{i}(\mathbf{r})\right|^{2}$. Being all bound states, they asymptotically decay to zero [205]. The slowest decay is of the highest occupied (ho) orbital, which therefore defines the decay 
rate of the density as a whole. This observation allows us to conclude that $\varphi_{\mathrm{ho}}(\mathbf{r}) \sim \chi(\mathbf{r}) \sim r^{\beta} e^{-\sqrt{2 I} r}$. Finally, the KS potential can be expressed in terms of the ho orbital as $v_{\mathrm{KS}}(\mathbf{r})=\varepsilon_{\mathrm{ho}}+\frac{1}{2}\left[\nabla^{2} \varphi_{\mathrm{ho}}(\mathbf{r})\right] / \varphi_{\mathrm{ho}}(\mathbf{r})$. Substitution of the asymptotic form of $\varphi_{\text {ho }}(\mathbf{r})$ into the above expression for $v_{\mathrm{KS}}(\mathbf{r})$ leads us to conclude that asymptotically $v_{\mathrm{KS}}(\mathbf{r}) \sim-(Z-(N-1)) / r$, as expected [206]. From here follows also the asymptotic behavior of the xc potential:

$$
v_{\mathrm{xc}}[n](\mathbf{r}) \rightarrow-\frac{1}{|\mathbf{r}|} .
$$

The asymptotic behavior of the KS and the KSP potentials is the same; the Pauli ingredient does not contribute at far distances. This conclusion is in agreement with the result of Ref. [109], Eq. (16), where an exact form for the Pauli potential is derived in terms of the KS energies, orbitals and the density.

To summarize, in this Section we reviewed the derivation for the asymptotic behavior of the exact xc energy density per particle, $e_{\mathrm{xc}}[n](\mathbf{r})$ (Eq. (13)) and of the exact xc potential, $v_{\mathrm{xc}}[n](\mathbf{r})$ (Eq. (16)), as $|\mathbf{r}| \rightarrow \infty$. The result for $e_{\mathrm{xc}}[n](\mathbf{r})$ has been obtained relying on the concepts of the adiabatic connection and of the xc hole, whereas for the result of $v_{\mathrm{xc}}[n](\mathbf{r})$ the exact electron factorization approach has been employed.

\section{EXCHANGE-CORRELATION POTENTIAL VIA THE EXCHANGE-CORRELATION HOLE}

In this section we further analyze the exact xc potential and its asymptotic behavior, combining the results of Sec. II A and Sec. II B.

The xc potential is, by definition, the functional derivative of the xc energy with respect to the density. We can therefore derive an expression for the xc potential starting from Eq. (11):

$$
v_{\mathrm{xc}}[n](\mathbf{r})=e_{\mathrm{xc}}[n](\mathbf{r})+\frac{1}{2} \iint d^{3} r^{\prime} d^{3} r^{\prime \prime} \frac{n\left(\mathbf{r}^{\prime}\right) \bar{\eta}_{\mathrm{xc}}\left(\mathbf{r} ; \mathbf{r}^{\prime \prime} \mid \mathbf{r}^{\prime}\right)}{\left|\mathbf{r}^{\prime}-\mathbf{r}^{\prime \prime}\right|}
$$

where $\bar{\eta}_{\mathrm{xc}}\left(\mathbf{r} ; \mathbf{r}^{\prime \prime} \mid \mathbf{r}^{\prime}\right):=\delta \bar{n}_{\mathrm{xc}}\left(\mathbf{r}^{\prime \prime} \mid \mathbf{r}^{\prime}\right) / \delta n(\mathbf{r})$ is the response of the $\lambda$-averaged xc hole to a density change at $\mathbf{r}$. Interestingly, the xc potential appears here as a sum of the xc energy density per particle and of an additional term, which is the electrostatic interaction between the ground-state density and the xc hole response, $\bar{\eta}_{\mathrm{xc}}$. From Eq. (17) we realize that whereas the asymptotic behavior of $e_{\mathrm{xc}}[n](\mathbf{r})$ directly follows from the sum rule for the xc hole (Eq. (12)), the asymptotic behavior of $v_{\mathrm{xc}}[n](\mathbf{r})$ depends not only on the properties of the xc hole as such, but also on the properties of its first derivative, $\bar{\eta}_{\mathrm{xc}}\left(\mathbf{r} ; \mathbf{r}^{\prime \prime} \mid \mathbf{r}^{\prime}\right)$. Therefore, the correct asymptotic behavior of $e_{\mathrm{xc}}$ and of $v_{\mathrm{xc}}$ (Eqs. (13) and (16)) can be viewed as two separate properties of the exact xc functional. When constructing approximations to exchange and correlation within DFT, this is a useful fact: it means that in principle correct asymptotics of $e_{\mathrm{xc}}$ does not guarantee correct asymptotics of $v_{\mathrm{xc}}$, and vice versa [71].
The xc hole response, $\bar{\eta}_{\mathrm{xc}}$, is a quantity that, to the best of my knowledge, did not receive yet much attention in the literature. Since it can be used to express the xc potential, knowledge of its properties is of high interest. One such property arises directly from the sum rule of the xc hole (Eq. (7)). By rewriting the sum rule for the general $\lambda$-case as $\int d^{3} r^{\prime \prime} n_{\mathrm{xc}}^{(\lambda)}\left(\mathbf{r}^{\prime \prime} \mid \mathbf{r}^{\prime}\right)=-1$ and operating with $\delta / \delta n(\mathbf{r})$ on both sides, one obtains

$$
\int d^{3} r^{\prime \prime} \eta_{\mathrm{xc}}^{(\lambda)}\left(\mathbf{r} ; \mathbf{r}^{\prime \prime} \mid \mathbf{r}^{\prime}\right)=0
$$

The same follows for $\bar{\eta}_{\mathrm{xc}}$, of course.

Another property one can derive is

$$
\iint d^{3} r^{\prime} d^{3} r^{\prime \prime} n\left(\mathbf{r}^{\prime}\right) \eta_{\mathrm{xc}}\left(\mathbf{r} ; \mathbf{r}^{\prime \prime} \mid \mathbf{r}^{\prime}\right)=0
$$

To prove Eq. (19), consider the quantity $g\left(\mathbf{r}^{\prime \prime}, \mathbf{r}^{\prime}\right)=$ $n\left(\mathbf{r}^{\prime}\right) n_{\mathrm{xc}}\left(\mathbf{r}^{\prime \prime} \mid \mathbf{r}^{\prime}\right)$. From Eq. (6), we find that $g\left(\mathbf{r}^{\prime \prime}, \mathbf{r}^{\prime}\right)=$ $\rho_{2}\left(\mathbf{r}^{\prime \prime}, \mathbf{r}^{\prime}\right)-n\left(\mathbf{r}^{\prime \prime}\right) n\left(\mathbf{r}^{\prime}\right)$. Next, from Eq. (5) we realize that $\iint d^{3} r^{\prime} d^{3} r^{\prime \prime} g\left(\mathbf{r}^{\prime \prime}, \mathbf{r}^{\prime}\right)=-N$ and therefore $\frac{\delta}{\delta n(\mathbf{r})} \iint d^{3} r^{\prime} d^{3} r^{\prime \prime} g\left(\mathbf{r}^{\prime \prime}, \mathbf{r}^{\prime}\right)=-1$. In parallel, $\delta g\left(\mathbf{r}^{\prime \prime}, \mathbf{r}^{\prime}\right) / \delta n(\mathbf{r})=n\left(\mathbf{r}^{\prime}\right) \eta_{\mathrm{xc}}\left(\mathbf{r} ; \mathbf{r}^{\prime \prime} \mid \mathbf{r}^{\prime}\right)+n_{\mathrm{xc}}\left(\mathbf{r}^{\prime \prime} \mid \mathbf{r}^{\prime}\right) \delta\left(\mathbf{r}-\mathbf{r}^{\prime}\right)$. Taking the double integral over the last expression and using the sum rule (7) yields Eq. (19).

A third property of the xc hole response function arises directly from combination of Eqs. (13), (16) and (17):

$$
\iint d^{3} r^{\prime} d^{3} r^{\prime \prime} \frac{n\left(\mathbf{r}^{\prime}\right) \bar{\eta}_{\mathrm{xc}}\left(\mathbf{r} ; \mathbf{r}^{\prime \prime} \mid \mathbf{r}^{\prime}\right)}{\left|\mathbf{r}^{\prime}-\mathbf{r}^{\prime \prime}\right|} \rightarrow-\frac{1}{|\mathbf{r}|}
$$

This expression does not imply directly the asymptotic $\mathbf{r}$-dependence of $\bar{\eta}_{\mathrm{xc}}\left(\mathbf{r} ; \mathbf{r}^{\prime \prime} \mid \mathbf{r}^{\prime}\right)$ itself. Further findings on the exchange $(\mathrm{x})$ part of $\bar{\eta}_{\mathrm{xc}}$ are presented in

\section{EXACT EXCHANGE HOLE RESPONSE FUNCTION}

In this section we make the first step in the direction of investigating the exact xc hole response function, $\eta_{\mathrm{xc}}\left(\mathbf{r} ; \mathbf{r}^{\prime \prime} \mid \mathbf{r}^{\prime}\right)$ and derive its exact-exchange (EXX) part. Due to the fact that the spin dependence of EXX is easily and transparently represented, the following derivation is performed within the spin-polarized version of KS-DFT.

The EXX energy functional is usually expressed as

$$
\begin{gathered}
E_{\mathrm{x}}^{\mathrm{exact}}=-\frac{1}{2} \sum_{\tau} \sum_{i=1}^{N_{\tau}} \sum_{j=1}^{N_{\tau}} \iint \frac{\varphi_{i \tau}^{*}\left(\mathbf{r}^{\prime}\right) \varphi_{j \tau}^{*}\left(\mathbf{r}^{\prime \prime}\right) \varphi_{i \tau}\left(\mathbf{r}^{\prime \prime}\right) \varphi_{j \tau}\left(\mathbf{r}^{\prime}\right)}{\left|\mathbf{r}^{\prime}-\mathbf{r}^{\prime \prime}\right|} \\
\times d^{3} r^{\prime} d^{3} r^{\prime \prime}
\end{gathered}
$$

where $\tau$ is the spin index, which takes the values $\uparrow$ or $\downarrow, \varphi_{i \tau}(\mathbf{r})$ are the KS spin-orbitals, being the eigenstates of the Schrödinger equation $\left(-\frac{1}{2} \nabla^{2}+v_{\mathrm{KS}, \tau}(\mathbf{r})\right) \varphi_{i \tau}(\mathbf{r})=$ $\varepsilon_{i \tau} \varphi_{i \tau}(\mathbf{r})$, and $N_{\tau}$ is the number of electrons in the $\tau$-spin-channel. It is assumed that the first $N_{\tau}$ low-lying 
spin-orbitals are occupied by one electron each and therefore introduction of occupation numbers is unnecessary in the present derivation.

Another way to express the EXX energy is via the KS one-electron reduced density matrix, $\rho_{1}\left(\mathbf{r}^{\prime \prime} \tau, \mathbf{r}^{\prime} \tau\right)[9]$, which is obtained from the KS many-electron wavefunction, $\Phi_{\mathrm{KS}}\left(\mathbf{r}_{1} \tau_{1}, \mathbf{r}_{2} \tau_{2} \cdots \mathbf{r}_{N} \tau_{N}\right)$, in the following way [10]:

$$
\begin{aligned}
& \rho_{1}\left(\mathbf{r}^{\prime \prime} \tau, \mathbf{r}^{\prime} \tau\right)=N \sum_{\tau_{2} \cdots \tau_{N}} \int d^{3} r_{2} \cdots \int d^{3} r_{N} \\
& \Phi_{\mathrm{KS}}^{*}\left(\mathbf{r}^{\prime \prime} \tau, \mathbf{r}_{2} \tau_{2} \cdots \mathbf{r}_{N} \tau_{N}\right) \Phi_{\mathrm{KS}}\left(\mathbf{r}^{\prime} \tau, \mathbf{r}_{2} \tau_{2} \cdots \mathbf{r}_{N} \tau_{N}\right) .
\end{aligned}
$$

Recalling that $\Phi_{\mathrm{KS}}$ is a Slater determinant constructed of KS spin-orbitals, we can simplify the expression for $\rho_{1}\left(\mathbf{r}^{\prime \prime} \tau, \mathbf{r}^{\prime} \tau\right)$ to

$$
\rho_{1}\left(\mathbf{r}^{\prime \prime} \tau, \mathbf{r}^{\prime} \tau\right)=\sum_{i=1}^{N_{\tau}} \varphi_{i \tau}^{*}\left(\mathbf{r}^{\prime \prime}\right) \varphi_{i \tau}\left(\mathbf{r}^{\prime}\right) .
$$

Noting that $\rho_{1}\left(\mathbf{r}^{\prime \prime} \tau, \mathbf{r}^{\prime} \tau\right)=\rho_{1}^{*}\left(\mathbf{r}^{\prime} \tau, \mathbf{r}^{\prime \prime} \tau\right)$, we express the EXX energy (21) as

$$
E_{\mathrm{x}}^{\text {exact }}=-\frac{1}{2} \sum_{\tau} \iint d^{3} r^{\prime} d^{3} r^{\prime \prime} \frac{\left|\rho_{1}\left(\mathbf{r}^{\prime \prime} \tau, \mathbf{r}^{\prime} \tau\right)\right|^{2}}{\left|\mathbf{r}^{\prime}-\mathbf{r}^{\prime \prime}\right|} .
$$

A third way to express the EXX energy is via the EXX hole, $n_{\mathrm{x}}\left(\mathbf{r}^{\prime \prime} \mid \mathbf{r}^{\prime}\right)$. To obtain the latter, one first finds the KS two-electron reduced density matrix, $\rho_{2}^{\mathrm{KS}}\left(\mathbf{r}^{\prime \prime}, \mathbf{r}^{\prime}\right)$, by substitution of $\Phi_{\mathrm{KS}}$ into Eq. (5). One then finds that $\rho_{2}^{\mathrm{KS}}\left(\mathbf{r}^{\prime \prime}, \mathbf{r}^{\prime}\right)=n\left(\mathbf{r}^{\prime \prime}\right) n\left(\mathbf{r}^{\prime}\right)-\sum_{\tau}\left|\rho_{1}\left(\mathbf{r}^{\prime \prime} \tau, \mathbf{r}^{\prime} \tau\right)\right|^{2}$. Then, following the line of thought of Sec. II A, one realizes that the EXX hole equals

$$
n_{\mathrm{x}}\left(\mathbf{r}^{\prime \prime} \mid \mathbf{r}^{\prime}\right)=-\frac{1}{n\left(\mathbf{r}^{\prime}\right)} \sum_{\tau}\left|\rho_{1}\left(\mathbf{r}^{\prime \prime} \tau, \mathbf{r}^{\prime} \tau\right)\right|^{2} .
$$

Subsequently, the EXX energy density per particle equals

$$
\begin{aligned}
e_{\mathrm{x}}^{\text {exact }}\left(\mathbf{r}^{\prime}\right) & =\frac{1}{2} \int d^{3} r^{\prime \prime} \frac{n_{\mathrm{x}}\left(\mathbf{r}^{\prime \prime} \mid \mathbf{r}^{\prime}\right)}{\left|\mathbf{r}^{\prime}-\mathbf{r}^{\prime \prime}\right|}= \\
& =-\frac{1}{2 n\left(\mathbf{r}^{\prime}\right)} \sum_{\tau} \int d^{3} r^{\prime \prime} \frac{\left|\rho_{1}\left(\mathbf{r}^{\prime \prime} \tau, \mathbf{r}^{\prime} \tau\right)\right|^{2}}{\left|\mathbf{r}^{\prime}-\mathbf{r}^{\prime \prime}\right|}
\end{aligned}
$$

and the EXX energy is expressed as

$$
E_{\mathrm{x}}^{\text {exact }}=\frac{1}{2} \iint d^{3} r^{\prime} d^{3} r^{\prime \prime} \frac{n\left(\mathbf{r}^{\prime}\right) n_{\mathrm{x}}\left(\mathbf{r}^{\prime \prime} \mid \mathbf{r}^{\prime}\right)}{\left|\mathbf{r}^{\prime}-\mathbf{r}^{\prime \prime}\right|}
$$

substitution of Eq. (25) into Eq. (27) leads to Eq. (24), as required. Using Eqs. (27) and (26), the EXX potential can be formally expressed as

$$
\begin{aligned}
v_{\mathrm{x}, \sigma}^{\text {exact }}(\mathbf{r})= & \frac{\delta E_{\mathrm{x}}^{\text {exact }}}{\delta n_{\sigma}(\mathbf{r})}=e_{\mathrm{x}}^{\text {exact }}(\mathbf{r})+ \\
& +\frac{1}{2} \iint d^{3} r^{\prime} d^{3} r^{\prime \prime} \frac{n\left(\mathbf{r}^{\prime}\right) \eta_{\mathrm{x}, \sigma}\left(\mathbf{r} ; \mathbf{r}^{\prime \prime} \mid \mathbf{r}^{\prime}\right)}{\left|\mathbf{r}^{\prime}-\mathbf{r}^{\prime \prime}\right|}
\end{aligned}
$$

where $\eta_{\mathrm{x}, \sigma}\left(\mathbf{r} ; \mathbf{r}^{\prime \prime} \mid \mathbf{r}^{\prime}\right)=\delta n_{\mathrm{x}}\left(\mathbf{r}^{\prime \prime} \mid \mathbf{r}^{\prime}\right) / \delta n_{\sigma}(\mathbf{r})$ is the response of the EXX hole, $n_{\mathrm{x}}\left(\mathbf{r}^{\prime \prime} \mid \mathbf{r}^{\prime}\right)$, to a variation is the $\sigma$-spindensity, $n_{\sigma}(\mathbf{r})$. Using Eq. (25), this response function can be written as

$$
\begin{aligned}
& \eta_{\mathrm{x}, \sigma}\left(\mathbf{r} ; \mathbf{r}^{\prime \prime} \mid \mathbf{r}^{\prime}\right)=-\frac{n_{\mathrm{x}}\left(\mathbf{r}^{\prime \prime} \mid \mathbf{r}^{\prime}\right)}{n(\mathbf{r})} \delta\left(\mathbf{r}-\mathbf{r}^{\prime}\right)- \\
& \quad-\frac{1}{n\left(\mathbf{r}^{\prime}\right)} \sum_{\tau}\left[\rho_{1}^{*}\left(\mathbf{r}^{\prime \prime} \tau, \mathbf{r}^{\prime} \tau\right) w_{\sigma}\left(\mathbf{r} ; \mathbf{r}^{\prime \prime}, \mathbf{r}^{\prime}, \tau\right)+c . c .\right]
\end{aligned}
$$

where $w_{\sigma}\left(\mathbf{r} ; \mathbf{r}^{\prime \prime}, \mathbf{r}^{\prime}, \tau\right):=\delta \rho_{1}\left(\mathbf{r}^{\prime \prime} \tau, \mathbf{r}^{\prime} \tau\right) / \delta n_{\sigma}(\mathbf{r})$ is the response of the KS one-electron reduced density matrix. The EXX hole response $\eta_{\mathrm{x}, \sigma}\left(\mathbf{r} ; \mathbf{r}^{\prime \prime} \mid \mathbf{r}^{\prime}\right)$ satisfies the sum rule for hole response functions (18): integrating over $\mathbf{r}^{\prime \prime}$, while remembering that $\int d^{3} r^{\prime \prime} n_{\mathrm{x}}\left(\mathbf{r}^{\prime \prime} \mid \mathbf{r}^{\prime}\right)=-1$, that $\int d^{3} r^{\prime \prime}\left|\rho_{1}\left(\mathbf{r}^{\prime \prime} \tau, \mathbf{r}^{\prime} \tau\right)\right|^{2}=n_{\tau}\left(\mathbf{r}^{\prime}\right)$ and that

$$
\begin{aligned}
& \int d^{3} r^{\prime \prime} \rho_{1}^{*}\left(\mathbf{r}^{\prime \prime} \tau, \mathbf{r}^{\prime} \tau\right) w_{\sigma}\left(\mathbf{r} ; \mathbf{r}^{\prime \prime}, \mathbf{r}^{\prime}, \tau\right)+c . c .= \\
& =\frac{\delta}{\delta n_{\sigma}(\mathbf{r})} \int d^{3} r^{\prime \prime}\left|\rho_{1}\left(\mathbf{r}^{\prime \prime} \tau, \mathbf{r}^{\prime} \tau\right)\right|^{2}=\delta_{\sigma \tau} \delta\left(\mathbf{r}-\mathbf{r}^{\prime}\right)
\end{aligned}
$$

one proves Eq. (18) for $\eta_{\mathrm{x}, \sigma}$. Furthermore, $\eta_{\mathrm{x}, \sigma}$ satisfies also Eq. (19), which can be proved in a similar manner. Consequently, also the exact correlation hole response function, $\eta_{\mathrm{c}, \sigma}$, has to satisfy Eqs. (18) and (19).

In the following we obtain an analytical expression for $\eta_{\mathrm{x}, \sigma}$ by deriving an expression for $w_{\sigma}$. The main challenge in deriving $w_{\sigma}$ as a density derivative of $\rho_{1}$ lies in the fact that $\rho_{1}$ is not explicitly density-dependent: as we see in Eq. (23), $\rho_{1}$ is explicitly expressed in terms of the KS spin-orbitals, which in turn depend on the spin densities, via the KS potential. Therefore, the dependence of $\rho_{1}$ on the density is implicit. This challenge is not unique to $\rho_{1}$ : the same issue raises when one wishes to obtain, for example, the EXX potential from the EXX energy. To find the density-derivative in such cases, we employ the wellknown OEP method [90]. Our case is complicated by the fact that $\rho_{1}$ is a complex quantity. The OEP formalism, originally designed to take the derivative of an energy - manifestly a real quantity - is generalized accordingly in the Appendix. Relying on Eq. (A.20) from the Appendix, we calculate $w_{\sigma}\left(\mathbf{r} ; \mathbf{r}^{\prime \prime}, \mathbf{r}^{\prime}, \tau\right)$ in the Krieger-LiIafrate (KLI) approximation [117], namely neglecting the orbitals shifts $\psi_{i \sigma}^{*}(\mathbf{r})$ and $\widetilde{\psi}_{i \sigma}(\mathbf{r})$ in Eq. (A.20). Within this approximation the response function $w_{\sigma}\left(\mathbf{r} ; \mathbf{r}^{\prime \prime}, \mathbf{r}^{\prime}, \tau\right)$ is expressed as

$$
\begin{aligned}
& w_{\sigma}^{\mathrm{KLI}}\left(\mathbf{r} ; \mathbf{r}^{\prime \prime}, \mathbf{r}^{\prime}, \tau\right)=\frac{1}{n_{\sigma}(\mathbf{r})}\left[\sum_{i=1}^{N_{\sigma}} U_{i \sigma}\left(\mathbf{r} ; \mathbf{r}^{\prime \prime}, \mathbf{r}^{\prime}, \tau\right)\left|\varphi_{i \sigma}(\mathbf{r})\right|^{2}+\right. \\
& \left.+\sum_{i=1}^{N_{\sigma}-1}\left(\bar{w}_{i \sigma}\left(\mathbf{r}^{\prime \prime}, \mathbf{r}^{\prime}, \tau\right)-\bar{U}_{i \sigma}\left(\mathbf{r}^{\prime \prime}, \mathbf{r}^{\prime}, \tau\right)\right)\left|\varphi_{i \sigma}(\mathbf{r})\right|^{2}\right],
\end{aligned}
$$


where

$$
\begin{aligned}
& U_{i \sigma}\left(\mathbf{r} ; \mathbf{r}^{\prime \prime}, \mathbf{r}^{\prime}, \tau\right)= \\
& =\frac{1}{2}\left(\frac{1}{\varphi_{i \sigma}^{*}(\mathbf{r})} \frac{\delta \rho_{1}\left(\mathbf{r}^{\prime \prime} \tau, \mathbf{r}^{\prime} \tau\right)}{\delta \varphi_{i \sigma}(\mathbf{r})}+\frac{1}{\varphi_{i \sigma}(\mathbf{r})} \frac{\delta \rho_{1}\left(\mathbf{r}^{\prime \prime} \tau, \mathbf{r}^{\prime} \tau\right)}{\delta \varphi_{i \sigma}^{*}(\mathbf{r})}\right)
\end{aligned}
$$

includes the derivatives of $\rho_{1}$ with respect to the KS orbitals and the bar above a quantity means its r-average with respect to the $i^{\text {th }} \mathrm{KS}$ orbital, namely, $\bar{w}_{i \sigma}\left(\mathbf{r}^{\prime \prime}, \mathbf{r}^{\prime}, \tau\right)=\int d^{3} r \varphi_{i \sigma}^{*}(\mathbf{r}) w_{\sigma}\left(\mathbf{r} ; \mathbf{r}^{\prime \prime}, \mathbf{r}^{\prime}, \tau\right) \varphi_{i \sigma}(\mathbf{r})$ and $\bar{U}_{i \sigma}\left(\mathbf{r}^{\prime \prime}, \mathbf{r}^{\prime}, \tau\right)=\int d^{3} r \varphi_{i \sigma}^{*}(\mathbf{r}) U_{i \sigma}\left(\mathbf{r} ; \mathbf{r}^{\prime \prime}, \mathbf{r}^{\prime}, \tau\right) \varphi_{i \sigma}(\mathbf{r})$ (see the Appendix for more details). The standard alignment convention $\bar{w}_{N_{\sigma} \sigma}\left(\mathbf{r}^{\prime \prime}, \mathbf{r}^{\prime}, \tau\right)=\bar{U}_{N_{\sigma} \sigma}\left(\mathbf{r}^{\prime \prime}, \mathbf{r}^{\prime}, \tau\right)$ is employed. From Eq. (23) we find that

$$
\begin{aligned}
& U_{i \sigma}\left(\mathbf{r} ; \mathbf{r}^{\prime \prime}, \mathbf{r}^{\prime}, \tau\right)= \\
& =\frac{\delta_{\sigma \tau}}{2}\left(\frac{\varphi_{i \sigma}^{*}\left(\mathbf{r}^{\prime \prime}\right)}{\varphi_{i \sigma}^{*}\left(\mathbf{r}^{\prime}\right)} \delta\left(\mathbf{r}-\mathbf{r}^{\prime}\right)+\frac{\varphi_{i \sigma}\left(\mathbf{r}^{\prime}\right)}{\varphi_{i \sigma}\left(\mathbf{r}^{\prime \prime}\right)} \delta\left(\mathbf{r}-\mathbf{r}^{\prime \prime}\right)\right)
\end{aligned}
$$

and $\bar{U}_{i \sigma}\left(\mathbf{r}^{\prime \prime}, \mathbf{r}^{\prime}, \tau\right)=\delta_{\sigma \tau} \varphi_{i \sigma}^{*}\left(\mathbf{r}^{\prime \prime}\right) \varphi_{i \sigma}\left(\mathbf{r}^{\prime}\right)$. Next, to find $\bar{w}_{i \sigma}\left(\mathbf{r}^{\prime \prime}, \mathbf{r}^{\prime}, \tau\right)$, we multiply both sides of Eq. (31) by $\left|\varphi_{j \sigma}(\mathbf{r})\right|^{2}$ and integrate over $\mathbf{r}$ :

$$
\begin{gathered}
\bar{w}_{j \sigma}\left(\mathbf{r}^{\prime \prime}, \mathbf{r}^{\prime}, \tau\right)=\frac{\delta_{\sigma \tau}}{2} \rho_{1}\left(\mathbf{r}^{\prime \prime} \sigma, \mathbf{r}^{\prime} \sigma\right)\left(\frac{\left|\varphi_{j \sigma}\left(\mathbf{r}^{\prime}\right)\right|^{2}}{n_{\sigma}\left(\mathbf{r}^{\prime}\right)}+\frac{\left|\varphi_{j \sigma}\left(\mathbf{r}^{\prime \prime}\right)\right|^{2}}{n_{\sigma}\left(\mathbf{r}^{\prime \prime}\right)}\right) \\
+\sum_{i=1}^{N_{\sigma}-1} m_{i j}^{\sigma}\left(\bar{w}_{i \sigma}\left(\mathbf{r}^{\prime \prime}, \mathbf{r}^{\prime}, \tau\right)-\bar{U}_{i \sigma}\left(\mathbf{r}^{\prime \prime}, \mathbf{r}^{\prime}, \tau\right)\right) .
\end{gathered}
$$

Here $m_{i j}^{\sigma}=\int d^{3} r\left|\varphi_{i \sigma}(\mathbf{r})\right|^{2}\left|\varphi_{j \sigma}(\mathbf{r})\right|^{2} / n_{\sigma}(\mathbf{r})$. Equation (34) can be conveniently represented in a vectormatrix form as

$$
\vec{s}=\vec{s}_{0}+M_{\sigma} \vec{s},
$$

if we define the vector $\vec{s}$ of length $\left(N_{\sigma}-1\right)$, whose elements are given as $s_{j}=\bar{w}_{j \sigma}\left(\mathbf{r}^{\prime \prime}, \mathbf{r}^{\prime}, \tau\right)-\bar{U}_{j \sigma}\left(\mathbf{r}^{\prime \prime}, \mathbf{r}^{\prime}, \tau\right)$, the vector $\vec{s}_{0}$, whose elements are given as

$$
\begin{gathered}
\left(s_{0}\right)_{j}=\delta_{\sigma \tau}\left[\frac{1}{2} \rho_{1}\left(\mathbf{r}^{\prime \prime} \sigma, \mathbf{r}^{\prime} \sigma\right)\left(\frac{\left|\varphi_{j \sigma}\left(\mathbf{r}^{\prime}\right)\right|^{2}}{n_{\sigma}\left(\mathbf{r}^{\prime}\right)}+\frac{\left|\varphi_{j \sigma}\left(\mathbf{r}^{\prime \prime}\right)\right|^{2}}{n_{\sigma}\left(\mathbf{r}^{\prime \prime}\right)}\right)-\right. \\
\left.-\varphi_{j \sigma}^{*}\left(\mathbf{r}^{\prime \prime}\right) \varphi_{j \sigma}\left(\mathbf{r}^{\prime}\right)\right]
\end{gathered}
$$

and the $\left(N_{\sigma}-1\right) \times\left(N_{\sigma}-1\right)$ matrix $M_{\sigma}$, whose elements are the above-defined integrals $m_{i j}^{\sigma}$. Equation (35) is then formally solved as $\vec{s}=\left(\mathbb{I}-M_{\sigma}\right)^{-1} \vec{s}_{0}$. This paves the way to analytically obtain the averages $\bar{w}_{i \sigma}$.

Proceeding with the derivation, we define the matrix $\widetilde{M}_{\sigma}:=\left(\mathbb{I}-M_{\sigma}\right)^{-1}$, and the elements of this matrix as $\widetilde{m}_{i j}^{\sigma}$. Given the KS orbitals $\varphi_{i \sigma}(\mathbf{r})$, these matrix elements can be numerically calculated with relatively little effort. Assuming $\widetilde{m}_{i j}^{\sigma}$ are given, we can now express the elements of vector $\vec{s}$ as

$$
\begin{aligned}
& s_{i}=\sum_{j=1}^{N_{\sigma}-1} \widetilde{m}_{i j}^{\sigma}\left(s_{0}\right)_{j}=\delta_{\sigma \tau}\left[\frac{1}{2} \rho_{1}\left(\mathbf{r}^{\prime \prime} \sigma, \mathbf{r}^{\prime} \sigma\right) \times\right. \\
& \times \sum_{j=1}^{N_{\sigma}-1} \widetilde{m}_{i j}^{\sigma}\left(\frac{\left|\varphi_{j \sigma}\left(\mathbf{r}^{\prime}\right)\right|^{2}}{n_{\sigma}\left(\mathbf{r}^{\prime}\right)}+\frac{\left|\varphi_{j \sigma}\left(\mathbf{r}^{\prime \prime}\right)\right|^{2}}{n_{\sigma}\left(\mathbf{r}^{\prime \prime}\right)}\right)- \\
& \\
&\left.-\sum_{j=1}^{N_{\sigma}-1} \tilde{m}_{i j}^{\sigma} \varphi_{j \sigma}^{*}\left(\mathbf{r}^{\prime \prime}\right) \varphi_{j \sigma}\left(\mathbf{r}^{\prime}\right)\right]
\end{aligned}
$$

Next, we insert Eq. (33) and (37) into Eq. (31) to explicitly express the response function $w_{\sigma}\left(\mathbf{r} ; \mathbf{r}^{\prime \prime}, \mathbf{r}^{\prime}, \tau\right)$ in the KLI approximation:

$$
\begin{aligned}
& w_{\sigma}^{\mathrm{KLI}}\left(\mathbf{r} ; \mathbf{r}^{\prime \prime}, \mathbf{r}^{\prime}, \tau\right)=\delta_{\sigma \tau}\left[\frac{1}{2} \rho_{1}\left(\mathbf{r}^{\prime \prime} \sigma, \mathbf{r}^{\prime} \sigma\right)\left(\frac{\delta\left(\mathbf{r}-\mathbf{r}^{\prime}\right)}{n_{\sigma}\left(\mathbf{r}^{\prime}\right)}+\frac{\delta\left(\mathbf{r}-\mathbf{r}^{\prime \prime}\right)}{n_{\sigma}\left(\mathbf{r}^{\prime \prime}\right)}\right)+\right. \\
& \left.+\frac{1}{2} \rho_{1}\left(\mathbf{r}^{\prime \prime} \sigma, \mathbf{r}^{\prime} \sigma\right) \sum_{i=1}^{N_{\sigma}-1} \sum_{j=1}^{N_{\sigma}-1} \widetilde{m}_{i j}^{\sigma}\left(\frac{\left|\varphi_{j \sigma}\left(\mathbf{r}^{\prime}\right)\right|^{2}}{n_{\sigma}\left(\mathbf{r}^{\prime}\right)}+\frac{\left|\varphi_{j \sigma}\left(\mathbf{r}^{\prime \prime}\right)\right|^{2}}{n_{\sigma}\left(\mathbf{r}^{\prime \prime}\right)}\right) \frac{\left|\varphi_{i \sigma}(\mathbf{r})\right|^{2}}{n_{\sigma}(\mathbf{r})}-\sum_{i=1}^{N_{\sigma}-1} \sum_{j=1}^{N_{\sigma}-1} \widetilde{m}_{i j}^{\sigma} \varphi_{j \sigma}^{*}\left(\mathbf{r}^{\prime \prime}\right) \varphi_{j \sigma}\left(\mathbf{r}^{\prime}\right) \frac{\left|\varphi_{i \sigma}(\mathbf{r})\right|^{2}}{n_{\sigma}(\mathbf{r})}\right]
\end{aligned}
$$

In the following we shortly analyze the achieved result. First, the response function $w_{\sigma}\left(\mathbf{r} ; \mathbf{r}^{\prime \prime}, \mathbf{r}^{\prime}, \tau\right)$ is diagonal with respect to $\sigma$ and $\tau$, meaning that variation in a given spin-density creates response of the KS one-electron reduced density matrix in that very spin channel, but not in the opposite one. For this reason, from now on we drop for brevity the argument $\tau$ in the expressions for $w_{\sigma}$. Second, the response function satisfies $w_{\sigma}\left(\mathbf{r} ; \mathbf{r}^{\prime \prime}, \mathbf{r}^{\prime}\right)=w_{\sigma}^{*}\left(\mathbf{r} ; \mathbf{r}^{\prime}, \mathbf{r}^{\prime \prime}\right)$, as expected. Third, from
Eq. (38) we realize that with respect to $\mathbf{r}$ the response function $w_{\sigma}$ has terms of two types: an extremely localized term, due to the presence of the Dirac deltafunctions, $\delta\left(\mathbf{r}-\mathbf{r}^{\prime}\right)$ and $\delta\left(\mathbf{r}-\mathbf{r}^{\prime \prime}\right)$, and a term that is relatively spread out, which decays exponentially with $\mathbf{r}$, due to the ratios $\left|\varphi_{i \sigma}(\mathbf{r})\right|^{2} / n_{\sigma}(\mathbf{r})$.

Finally, we are ready to obtain the result for the EXX hole response function, $\eta_{\mathrm{x}, \sigma}\left(\mathbf{r} ; \mathbf{r}^{\prime \prime} \mid \mathbf{r}^{\prime}\right)$, by substituting Eqs. (38) and (25) into Eq. (29): 


$$
\begin{aligned}
& \eta_{\mathrm{x}, \sigma}^{\mathrm{KLI}}\left(\mathbf{r} ; \mathbf{r}^{\prime \prime} \mid \mathbf{r}^{\prime}\right)=-\frac{1}{n\left(\mathbf{r}^{\prime}\right)}\left\{\frac{\left|\rho_{1}\left(\mathbf{r}^{\prime \prime} \sigma, \mathbf{r}^{\prime} \sigma\right)\right|^{2}}{n_{\sigma}\left(\mathbf{r}^{\prime \prime}\right)} \delta\left(\mathbf{r}-\mathbf{r}^{\prime \prime}\right)+\frac{\left|\rho_{1}\left(\mathbf{r}^{\prime \prime} \sigma, \mathbf{r}^{\prime} \sigma\right)\right|^{2}}{n_{\sigma}\left(\mathbf{r}^{\prime}\right)} \delta\left(\mathbf{r}-\mathbf{r}^{\prime}\right)-\frac{\sum_{\tau}\left|\rho_{1}\left(\mathbf{r}^{\prime \prime} \tau, \mathbf{r}^{\prime} \tau\right)\right|^{2}}{n\left(\mathbf{r}^{\prime}\right)} \delta\left(\mathbf{r}-\mathbf{r}^{\prime}\right)+\right. \\
& \left.+\sum_{i=1}^{N_{\sigma}-1} \sum_{j=1}^{N_{\sigma}-1} \widetilde{m}_{i j}^{\sigma}\left[\left|\rho_{1}\left(\mathbf{r}^{\prime \prime} \sigma, \mathbf{r}^{\prime} \sigma\right)\right|^{2}\left(\frac{\left|\varphi_{j \sigma}\left(\mathbf{r}^{\prime}\right)\right|^{2}}{n_{\sigma}\left(\mathbf{r}^{\prime}\right)}+\frac{\left|\varphi_{j \sigma}\left(\mathbf{r}^{\prime \prime}\right)\right|^{2}}{n_{\sigma}\left(\mathbf{r}^{\prime \prime}\right)}\right)-\left(\rho_{1}^{*}\left(\mathbf{r}^{\prime \prime} \sigma, \mathbf{r}^{\prime} \sigma\right) \varphi_{j \sigma}^{*}\left(\mathbf{r}^{\prime \prime}\right) \varphi_{j \sigma}\left(\mathbf{r}^{\prime}\right)+c . c .\right)\right] \frac{\left|\varphi_{i \sigma}(\mathbf{r})\right|^{2}}{n_{\sigma}(\mathbf{r})}\right\}
\end{aligned}
$$

Equation (39) is the central result derived in this section. Similarly to Eq. (38), this expression also includes extremely localized terms with delta-functions (first line of Eq. (39)) and somewhat delocalized terms, with the ratios $\left|\varphi_{i \sigma}(\mathbf{r})\right|^{2} / n_{\sigma}(\mathbf{r})$ (second line). Substitution of Eq. (39) into Eq. (28) yields, after some algebraic manipulations, the EXX potential in the KLI approximation [90], as required. Furthermore, $\eta_{\mathrm{x}, \sigma}^{\mathrm{KLI}}\left(\mathbf{r} ; \mathbf{r}^{\prime \prime} \mid \mathbf{r}^{\prime}\right)$ satisfies Eq. (19).

As to the contribution of $\eta_{\mathrm{x}, \sigma}^{\mathrm{KLI}}$ to the asymptotic behavior of the potential, which was our central concern in Sec. II B, we reach the following interesting finding: as we substitute Eq. (39) into Eq. (20), we find that the only terms of $\eta_{\mathrm{x}, \sigma}^{\mathrm{KLI}}$ that are significant for the asymptotic behavior are those extremely localized terms that appear in the first line of Eq. (39). Summed up, they give the correct $-1 /|\mathbf{r}|$ behavior. The delocalized terms that appear in the second line, and are proportional to $\left|\varphi_{i \sigma}(\mathbf{r})\right|^{2} / n_{\sigma}(\mathbf{r})$, decay exponentially, and therefore do not contribute asymptotically. In addition, it follows that those terms that are absent in the KLI approximation, but do appear in the full OEP treatment, do not contribute to the asymptotic behavior either (cf. Ref. [118] for an analogous conclusion for the potential). This finding is relevant when constructing approximations to the exchange functional via the exchange hole, $n_{\mathrm{x}}\left(\mathbf{r}^{\prime \prime} \mid \mathbf{r}^{\prime}\right)$, and its response, $\eta_{\mathrm{x}, \sigma}\left(\mathbf{r} ; \mathbf{r}^{\prime \prime} \mid \mathbf{r}^{\prime}\right)$ : for the aim of getting the correct $-1 /|\mathbf{r}|$ asymptotic behavior, one can focus on approximating those extremely localized terms of $\eta_{\mathrm{x}, \sigma}$ that appear in the first line of Eq. (39). As to the complementary correlation term, $\bar{\eta}_{\mathrm{c}, \sigma}$, it follows that the correlation contribution, $\iint d^{3} r^{\prime} d^{3} r^{\prime \prime} n\left(\mathbf{r}^{\prime}\right) \bar{\eta}_{\mathrm{c}, \sigma}\left(\mathbf{r} ; \mathbf{r}^{\prime \prime} \mid \mathbf{r}^{\prime}\right) /\left|\mathbf{r}^{\prime}-\mathbf{r}^{\prime \prime}\right|$ has to decay at far $\mathbf{r}$ slower than $1 /|\mathbf{r}|$, in order to satisfy Eq. (20).

\section{ASYMPTOTIC BEHAVIOR OF COMMON EXCHANGE-CORRELATION APPROXIMATIONS}

So far we described the asymptotic behavior of the $e x$ act xc energy density per particle and the corresponding exact potential. It is therefore appropriate to examine the performance of common approximations with respect to the expected exact results.

It has become popular to categorize density functional approximations (DFAs) according to the "Jacob's ladder" scheme originally introduced in Ref. [25] [207]. The ladder, which is schematically depicted in Fig. 1, consists of five rungs that help to climb from the inaccurate 'Hartree world' to the 'heaven of chemical accuracy'. The DFAs are distributed on the rungs of the ladder according to the sophistication of the ingredients used in their construction. Thus, on the lowest, first rung we find the local density approximation (LDA) [119, 120], whose energy density per particle is a function (not a functional!) only of the density $n(\mathbf{r})$ itself. On the second rung we find the GGAs, which depend also on the gradient of the density, $\nabla n$. Among them is the celebrated PBE [38] functional. On the third rung reside the meta-GGAs (e.g., [39, 121]). For them the use of second derivatives of the density, e.g. $\nabla^{2} n$, as well as of the quantity $\tau(\mathbf{r})=\frac{1}{2} \sum_{\sigma} \sum_{i=1}^{N_{\sigma}}\left|\nabla \varphi_{i \sigma}(\mathbf{r})\right|^{2}$ (which is the KS kinetic energy density), is allowed. On the fourth rung we find functionals that explicitly depend on KS orbitals, but only on the occupied ones. Among them are the EXX functional, global hybrid functionals, like PBEh [122-124] and B3LYP [125, 126], local hybrids, e.g., [64, 96, 127-130] and range-separated hybrids (RSHs) (see [78, 79, 131, 132] and references therein). The last, fifth rung hosts functionals that depend also on unoccupied KS orbitals and on orbital energies, $\varepsilon_{i}$. One example of such a functional is the random-phaseapproximation (RPA) [133]. The accuracy of DFAs is expected to improve the higher their rung on the ladder is. This happens normally at the price of increased complexity of the functional, of its numerical implementation and of the numerical effort required in calculations.

In the following, we review the asymptotic performance of DFAs on each rung of the ladder. On the first rung, the LDA exchange yields $e_{\mathrm{x}}^{\mathrm{LDA}}(\mathbf{r})=-\frac{3}{4}\left(\frac{3}{\pi}\right)^{1 / 3} n^{1 / 3}(\mathbf{r})$, and therefore $v_{\mathrm{x}}^{\mathrm{LDA}}(\mathbf{r})=-\left(\frac{3}{\pi}\right)^{1 / 3} n^{1 / 3}(\mathbf{r})$ [9]. Asymptotically, the density decays exponentially, and so do $e_{\mathrm{x}}^{\mathrm{LDA}}$ and $v_{\mathrm{x}}^{\mathrm{LDA}}$. For LDA correlation the asymptotic result is similar, with both $e_{\mathrm{c}}^{\mathrm{LDA}}$ and $v_{\mathrm{c}}^{\mathrm{LDA}}$ decaying as $n^{1 / 3}(\mathbf{r})$. This too rapid decay, which is characteristic of the LDA, is held responsible for many of its failures, e.g., inability to produce bound anions and poor prediction of the IP via $\varepsilon_{\text {ho }}$.

On the second and third rung the asymptotic performance of GGAs and meta-GGAs (also called semilocal approximations) is typically similar to that of LDA: the decay is exponential and therefore too rapid. Different are the Becke (B88) [134] and the ArmientoKümmel (AK13) [135] exchange functionals. In the for- 


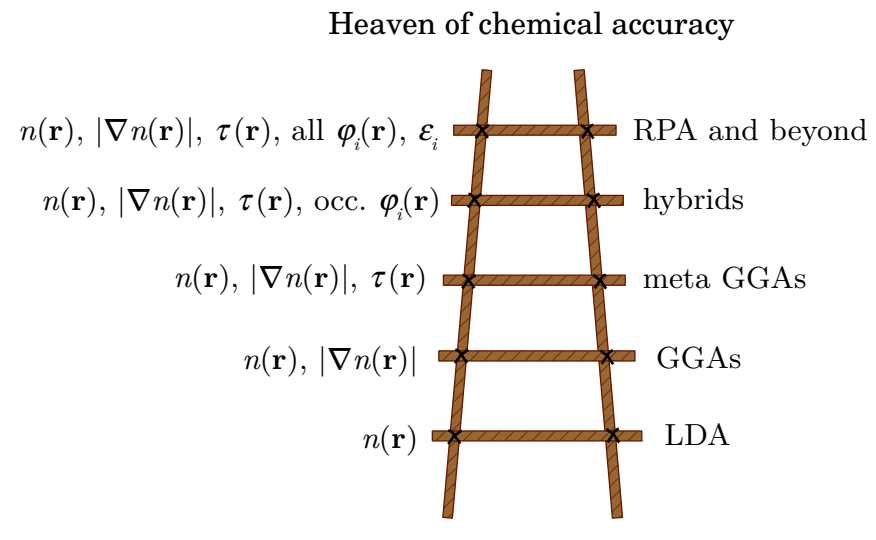

Hartree world

FIG. 1: "Jacob's ladder" of density functional approximations to the xc energy [25].

mer, $e_{\mathrm{x}}^{\mathrm{B} 88}(\mathbf{r})$ decays as $\sim 1 /(2|\mathbf{r}|)$, by construction (for an exponentially decaying density), but $v_{\mathrm{x}}^{\mathrm{B} 88}(\mathbf{r})$ does not produce the correct asymptotic limit [41]. In the latter, $v_{\mathrm{x}}^{\mathrm{AK} 13}(\mathbf{r})$ decays correctly, by construction, but $e_{\mathrm{x}}^{\mathrm{AK} 13}(\mathbf{r})$ does not.

The functional that clearly yields the correct asymptotic behavior for both $e_{\mathrm{xc}}(\mathbf{r})$ and $v_{\mathrm{xc}}(\mathbf{r})$ resides on the fourth rung. This is the EXX functional. The asymptotic behavior for $e_{\mathrm{x}}^{\text {exact }}(\mathbf{r})$ can be readily deduced from Eq. (26) by substituting $\mathbf{r}^{\prime}$ with $\mathbf{r}$ and taking the limit $\left|\mathbf{r}-\mathbf{r}^{\prime \prime}\right| \rightarrow|\mathbf{r}|$. Recalling that $\int d^{3} r^{\prime \prime} n_{\mathrm{x}}\left(\mathbf{r}^{\prime \prime} \mid \mathbf{r}\right)=-1$, it follows that $e_{\mathrm{x}}^{\text {exact }}(\mathbf{r}) \sim-1 /(2|\mathbf{r}|)$. To find the asymptotic behavior of $v_{\mathrm{x}, \sigma}^{\text {exact }}(\mathbf{r})$ we need to address the latter quantity with the OEP method. We do not present this derivation here, but only refer to a property of the OEP equation $[90,118]$ : the asymptotic behavior of $v_{\mathrm{x}, \sigma}^{\text {exact }}(\mathbf{r})$ is governed by the ho orbital-specific derivative

$$
\begin{aligned}
u_{N_{\sigma}, \sigma}(\mathbf{r}) & =\frac{1}{\varphi_{N_{\sigma}, \sigma}^{*}(\mathbf{r})} \frac{\delta E_{\mathrm{x}}^{\mathrm{exact}}}{\delta n_{\sigma}(\mathbf{r})}= \\
& =-\frac{1}{\varphi_{N_{\sigma}, \sigma}^{*}(\mathbf{r})} \sum_{j=1}^{N_{\sigma}} \varphi_{j \sigma}^{*}(\mathbf{r}) \int d^{3} r^{\prime} \frac{\varphi_{N_{\sigma}, \sigma}^{*}\left(\mathbf{r}^{\prime}\right) \varphi_{j \sigma}\left(\mathbf{r}^{\prime}\right)}{\left|\mathbf{r}-\mathbf{r}^{\prime}\right|}
\end{aligned}
$$

In the limit $\left|\mathbf{r}-\mathbf{r}^{\prime}\right| \rightarrow|\mathbf{r}|$, the latter quantity decays as $\sim 1 /|\mathbf{r}|$, and so does $v_{\mathrm{x}, \sigma}^{\text {exact }}(\mathbf{r})$. Therefore, EXX and also a combination of EXX and any (semi-)local correlation approximation perfectly perform in the asymptotic regime, both for $e_{\mathrm{xc}}(\mathbf{r})$ and $v_{\mathrm{xc}}(\mathbf{r})$. The main problem, however, is that bare EXX is known for its poor description of binding energies and structural properties $[10, \mathrm{ch}$. $2],[11]$, whereas adding a semi-local correlation functional can make performance even worse, leading to results inferior to those of a semi-local xc approximations. This is known as the incompatibility of EXX with (semi-)local correlation [90, Sec. III.D].

As a possible remedy to the above-described situation, one can consider a (global) hybrid DFA, which typically has the form

$$
E_{\mathrm{xc}}^{\mathrm{gl} . h y b}=(1-a) E_{\mathrm{x}}^{\mathrm{exact}}+a E_{\mathrm{x}}^{\mathrm{s} .-\mathrm{l} .}+E_{\mathrm{c}}^{\mathrm{s} .-\mathrm{l} .} .
$$

Here the superscript gl.hyb. stands for global hybrid, the superscript s.-l. stands for semi-local, and $a$ is a parameter between 0 and 1 [208]. Hybrid DFAs proved to be very successful in prediction of various properties and have nowadays a broad range of applications (see, e.g., [67, 90, 136] and references therein). However, global hybrids fail to produce the correct asymptotics for both $e_{\mathrm{xc}}(\mathbf{r})$ and $v_{\mathrm{xc}}(\mathbf{r})$. Assuming that the semi-local exchange and correlation terms decay exponentially, it follows that $e_{\mathrm{xc}}^{\text {gl.hyb. }}(\mathbf{r}) \sim-(1-a) /(2|\mathbf{r}|)$ and $v_{\mathrm{xc}}^{\text {gl.hyb. }}(\mathbf{r}) \sim-(1-a) /|\mathbf{r}|$, namely the resulting decay is too shallow by a factor of $(1-a)$, and the correct result is obtained only for $a=0$, i.e., for bare EXX plus semi-local correlation.

A further step towards construction of a correlation functional compatible with EXX has been made by introduction of local hybrids [127, 128]. Being a generalization of the global hybrid approach, a local hybrid xc approximation is defined via its energy density per particle:

$$
e_{\mathrm{xc}}^{\text {loc.hyb. }}(\mathbf{r})=(1-f(\mathbf{r})) e_{\mathrm{x}}^{\text {exact }}(\mathbf{r})+f(\mathbf{r}) e_{\mathrm{x}}^{\mathrm{s} .-\mathrm{l} .}(\mathbf{r})+e_{\mathrm{c}}^{\mathrm{s} .-\mathrm{l} .}(\mathbf{r}),
$$

being a linear combination of EXX and semi-local exchange, plus a semi-local correlation. Notably, the combination is not global, but local, due to the r-dependence of the function $f(\mathbf{r})$, termed the local mixing function (LMF). The flexibility introduced by the LMF allows, for example, to employ a higher portion of EXX in certain spatial regions of the system (e.g., where there is essentially one electron only), and a lower portion of EXX, hence a higher portion of semi-local exchange in other regions (e.g., where the density is close to a uniform one). A judicial choice of $f(\mathbf{r})$, which is a functional of the density, is in the heart of the local hybrid approach.

A good strategy is to design $f(\mathbf{r})$ by satisfying exact constraints $[64,129]$. One such constraint is related to the asymptotic limit, $|\mathbf{r}| \rightarrow \infty$. In this limit, we wish $e_{\mathrm{xc}}^{\text {loc.hyb. }}$ to be dominated by the EXX term. Assuming that the semi-local exchange and correlation terms of Eq. (42) decay exponentially (or at least faster than $1 /|\mathbf{r}|$ ), we realize that

$$
\lim _{|\mathbf{r}| \rightarrow \infty} f[n](\mathbf{r})=0
$$

is the LMF behavior we are looking for (see, for example, the LMF developed for the ISOcc local hybrid in Ref. [129]). If Eq. (43) is satisfied, $e_{\mathrm{xc}}^{\text {loc.hyb. }}(\mathbf{r}) \sim$ $(1-f(\mathbf{r})) e_{\mathrm{x}}^{\text {exact }}(\mathbf{r}) \sim-1 /(2|\mathbf{r}|)$, as required. But what about the corresponding potential, $v_{\mathrm{xc}}^{\text {loc.hyb. }}(\mathbf{r})$ ? One could naïvely expect from Eq. (42) that, by analogy to global hybrids, the potential will share with $e_{\mathrm{xc}}^{\text {loc.hyb. }}$ the same coefficient in the decay rate, and therefore behave as $-1 /|\mathbf{r}|$. However, a careful analysis performed in Ref. [71] has shown that $v_{\mathrm{xc}}^{\text {loc.hyb. }}(\mathbf{r}) \sim-\gamma /|\mathbf{r}|$, where 
$\gamma=1-\frac{1}{2} \int d^{3} r f(\mathbf{r})\left|\varphi_{\mathrm{ho}}(\mathbf{r})\right|^{2} \cdot \gamma$ is a system-specific quantity, and it can be shown that it always lies in the ragne $\left(\frac{1}{2}, 1\right]$. The desired value of 1 can be reached only by setting $f(\mathbf{r})=0$ everywhere in space, basically going back to the EXX functional. The obtained asymptotic decay of $-\gamma /|\mathbf{r}|$ is of course better than the rapid exponential decay of semi-local functionals, being closer to the exact one. Still, the finding of Ref. [71] implies that even such a broad and flexible family of functionals as local hybrids cannot generally yield the exact asymptotic behavior of $e_{\mathrm{xc}}(\mathbf{r})$ and $v_{\mathrm{xc}}(\mathbf{r})$ simultaneously. Therefore, an broader class of functionals has to be suggested.

\section{ASYMPTOTIC BEHAVIOR AND SELF-INTERACTION ERROR}

Incorrect asymptotic behavior is frequently associated in the the literature with the spurious self-interaction that plagues many DFAs. The notion of one-electron selfinteraction (SI) [43] emerges from the fact that for oneelectron systems (such as the $\mathrm{H}$ atom, the $\mathrm{H}_{2}^{+}$molecule, etc.) we know the KS potential and total energy exactly. For these cases, the only existing electron feels just the external potential. For this to happen, the exchange potential has to cancel out the Hartree potential and the correlation potential has to equal zero. The same relation is true for the corresponding energies [10]. In a DFA that does not satisfy the above criterion, the electron feels a spurious repulsion from itself. As a result, it tends to delocalize in space and the energy of the system is systematically too high. For example, the total energy for the $\mathrm{H}$ atom within the LSDA is higher by $4 \%$ comparing to the exact value (see e.g. [137, 138]). In a more general way, for any many-electron system, a DFA is considered [43] one-electron SI-free if

$$
E_{\mathrm{H}}\left[\left|\varphi_{i \sigma}(\mathbf{r})\right|^{2}\right]+E_{\mathrm{xc}}\left[\left|\varphi_{i \sigma}(\mathbf{r})\right|^{2}\right]=0
$$

where $\varphi_{i \sigma}(\mathbf{r})$ are the occupied KS spin-orbitals of the system. The definition above is not unique; see, e.g., the discussion in Ref. [139].

The SI problem is associated with problems of orbital delocalization [43, 140-143], ionization processes [144-147], charge transfer [148-151], dissociation of molecules [65, 152-158] and the interpretability of eigenvalues and orbitals e.g. in the context of photoemmission spectroscopy [80, 159-163].

Intuitively, there is a reason to connect self-interaction and wrong asymptotic behavior of the xc potential. Recall the intuitive explanation that we gave for the behavior of the potential in Sec. II B: located far away from a system, an electron in the exact KS potential feels the attraction of the nucleus screened by all the other $N-1$ electrons. In an approximate potential, the electron "interacts with itself", hence the behavior of the xc potential is wrong.

Furthermore, a strong association between selfinteraction and wrong asymptotics may have also

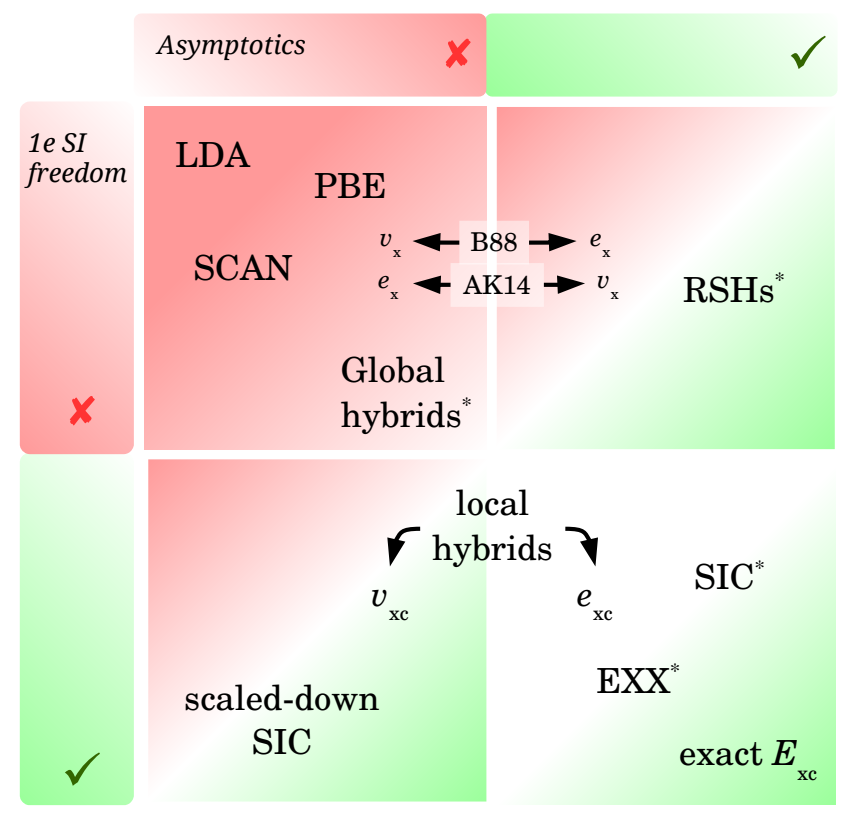

FIG. 2: One-electron self-interaction freedom versus correct asymptotic behavior of selected DFAs.

emerged from the fact that both the well-known EXX functional and the self-interaction-corrected (SIC) functional [43] (built on satisfying criterion (44)), have both properties right: they are one-electron self-interactionfree and the asymptotics of their potentials is correct [208].

However, it follows from Ref. [71], and further supported by the results of Refs. [151, 163], that (i) freedom from one-electron self-interaction does not necessarily lead to (ii) correct asymptotics of the xc potential, or the other way around. In fact, (i) and (ii) are two distinct properties of the exact xc functional. This work makes a further distinction: (iii) correct asymptotics of the xc energy-density per particle is yet another independent property of the exact functional; particularly, (iii) is distinct from (ii). These aforementioned properties can be regarded as three separate requirements that we wish an approximate xc functional to satisfy.

The matter is summarized in Fig. 2, which presents a $2 \times 2$ table indicating satisfaction of one-electron selfinteraction freedom (property (i); vertical) and correct asymptotics (properties (ii) and (iii) combined; horizontal). Each DFA can be positioned in this table according to the extent it satisfies the aforementioned properties.

Thus, in the top-left quadrant (SI present, wrong asymptotics) one finds the LDA, most GGAs, most metaGGAs and global hybrids (for DFAs marked in the figure with an asterisk, see footnote [208]). The latter are positioned closer to the right-bottom corner of this quadrant, as their asymptotic behavior goes at least with the correct power of $|\mathbf{r}|$, namely $|\mathbf{r}|^{-1}$, albeit with a wrong coefficient. Furthermore, global hybrids can also be viewed 'partly' SI-free; both these properties emerge from the 
presence of a fraction of EXX in the functional. Notably, there exist at least two semi-local DFAs, which were mentioned above, that reside on the border of the two upper quadrants: these are the B88 and the AK13 exchange functionals. Not being generally SI-free, these functionals yields the correct asymptotic behavior for either $e_{\mathrm{x}}(\mathbf{r})$ (B88) or $v_{\mathrm{x}}(\mathbf{r})(\mathrm{AK} 13)$, which is denoted in the table.

The bottom-right quadrant of the table (SI-free, correct asymptotics) hosts the EXX functional, the SIC functionals (implemented self-consistently within the KS scheme [139]) and of course the (unknown) exact xc functional. Local hybrid functionals (e.g., the ISOcc functional [129]) are located on the border of the two bottom quadrants. By an appropriate construction of the LMF, they can be SI-free and yield the correct asymptotic behavior of $e_{\mathrm{xc}}(\mathbf{r})$, but not of $v_{\mathrm{xc}}(\mathbf{r})$, as discussed in Sec. V.

Two additional classes of DFAs appear in Fig. 2. Scaled-down SIC functionals [164], located in the leftbottom quadrant, is SI-free, but its asymptotics is in error, due to the scale-down factor (see Ref. [164], Sec. V.B). Finally, RSHs have, by construction, the correct asymptotics, but are generally not SI-free, which determines their location in the top-right quadrant.

In summary, we conclude from Fig. 2 that various DFAs may appear be contaminated by one-electron selfinteraction or be SI-free and in parallel may possess correct asymptotics, for both $e_{\mathrm{xc}}(\mathbf{r})$ and $v_{\mathrm{xc}}(\mathbf{r})$, for one of them or for neither. All combinations exist among known DFAs, which shows that properties (i), (ii) and (iii) are distinct properties of the exact xc functional.

\section{SUMMARY AND OUTLOOK}

In this work we addressed the asymptotic behavior of the exchange-correlation potential, $v_{\mathrm{xc}}[n](\mathbf{r})$, and of the exchange-correlation energy density per particle, $e_{\mathrm{xc}}[n](\mathbf{r})$, as $|\mathbf{r}| \rightarrow \infty$, namely far away from a given finite system. Despite the high importance of satisfying exact constraints and despite the particular significance of having correct asymptotics in xc approximations, one has to admit that simultaneously obtaining the correct decay of both $e_{\mathrm{xc}}(\mathbf{r})$ and $v_{\mathrm{xc}}(\mathbf{r})$ is not an easy task at all. Not only semi-local approximations or global hybrids generally fail this task, but also such a large and flexible family of approximations as local hybrid functionals is not capable, in principle, to capture both these properties at once.

This article tried to contribute to the understanding why this is so. Indeed, while investing significant efforts in modelling $e_{\mathrm{xc}}(\mathbf{r})$, or in the case of local hybrids - modelling $e_{\mathrm{c}}(\mathbf{r})$ (compatible with $e_{\mathrm{x}}^{\text {exact }}(\mathbf{r})$ ), the xc potential, $v_{\mathrm{xc}}(\mathbf{r})$, directly result from $e_{\mathrm{xc}}(\mathbf{r})$ obtained by differentiation, without us having a direct influence on its properties in the process of approximation construction. Evidently, not all exact properties inherent to $e_{\mathrm{xc}}(\mathbf{r})$ naturally migrate to $v_{\mathrm{xc}}(\mathbf{r})$, as one would have naïvely hoped. As shown in Sec. III, asymptotic behavior of $e_{\mathrm{xc}}(\mathbf{r})$ and of $v_{\mathrm{xc}}(\mathbf{r})$ are two distinct properties of the exact xc func- tional, that do not follow one from the other. In particular, whereas the behavior of $e_{\mathrm{xc}}(\mathbf{r})$ is a direct consequence of the xc hole sum rule, the behavior of $v_{\mathrm{xc}}(\mathbf{r})$ depends on an additional quantity - the $\lambda$-averaged xc hole response function, $\bar{\eta}_{\mathrm{xc}}\left(\mathbf{r} ; \mathbf{r}^{\prime \prime}, \mathbf{r}^{\prime}\right)$.

The xc hole response function $\bar{\eta}_{\mathrm{xc}}\left(\mathbf{r} ; \mathbf{r}^{\prime \prime}, \mathbf{r}^{\prime}\right)$ was studied in Sec. III, with three of its exact properties analytically derived. Furthermore, in Sec. IV the exact exchange part of this response function, $\eta_{\mathrm{x}, \sigma}\left(\mathbf{r} ; \mathbf{r}^{\prime \prime}, \mathbf{r}^{\prime}\right)$, was obtained employing the OEP method (which has been generalized for this task, to address complex quantities (see Appendix)). In course of the derivation, the KLI approximation was employed and those terms of $\eta_{\mathrm{x}, \sigma}\left(\mathbf{r} ; \mathbf{r}^{\prime \prime}, \mathbf{r}^{\prime}\right)$ that contribute to the asymptotic behavior of the xc potential were identified. It was shown that other terms, including those that are beyond the KLI approximation, are not significant asymptotically and hence the KLI approximation is justified in our case. In addition, during the derivation of $\bar{\eta}_{\mathrm{xc}}\left(\mathbf{r} ; \mathbf{r}^{\prime \prime}, \mathbf{r}^{\prime}\right)$ we obtained $w_{\sigma}\left(\mathbf{r} ; \mathbf{r}^{\prime \prime}, \mathbf{r}^{\prime}\right)-$ the response of the KS one-electron density matrix, which is another quantity of interest in its own right.

Further progress in exploring $\eta_{\mathrm{x}, \sigma}\left(\mathbf{r} ; \mathbf{r}^{\prime \prime}, \mathbf{r}^{\prime}\right)$ can take several directions. First, going beyond the KLI approximation is desirable. Although not of direct significance to the questions of asymptotics, a more exact expression for $\eta_{\mathrm{x}, \sigma}\left(\mathbf{r} ; \mathbf{r}^{\prime \prime}, \mathbf{r}^{\prime}\right)$ can contribute to modelling the xc potential and reveal some of its exact properties. Second, generalization of the present approach to systems with a varying number of electrons, $N$, and subsequent derivation of $\eta_{\mathrm{x}, \sigma}\left(\mathbf{r} ; \mathbf{r}^{\prime \prime}, \mathbf{r}^{\prime}\right)$ and other quantities for a fractional $N$ can yield a better understanding of the exact xc potential in this scenario. Although systems with fractional $N$ are outside the scope of the present work, their treatment and detailed understanding of their properties is a topic of high significance [67, 72, 83-86, 136, 157, 165-173]. Finally, additional effort is required to study the properties of the correlation ingredient of the xc hole response function, $\eta_{\mathrm{c}, \sigma}\left(\mathbf{r} ; \mathbf{r}^{\prime \prime}, \mathbf{r}^{\prime}\right)$. This may be partly achieved via derivation of additional exact properties, following Eqs. (18), (19) and (20).

In conclusion of this work, it is appropriate to discuss the possible routes one can take aiming at new xc approximations that have correct asymptotics, simultaneously for $v_{\mathrm{xc}}[n](\mathbf{r})$ and $e_{\mathrm{xc}}[n](\mathbf{r})$, along with additional important properties, such as freedom from self-interaction, uniform scaling, size consistency, satisfying the IP theorem, and more. One such route is to directly model the xc potential, making it to satisfy the correct $\sim-1 /|\mathbf{r}|$ asymptotics as a constraint. Fruitful efforts in this direction took place over the years (see, e.g., [75, 174-177]) and the resulting approximations are of practical use. However, recovering the corresponding energy density per particle for a directly designed potential expression is not feasible, and the mathematical question of existence of an energy functional for a given potential stands. Therefore, the use of direct potential approximations is necessarily limited [178-180].

Another route towards approximations with correct 


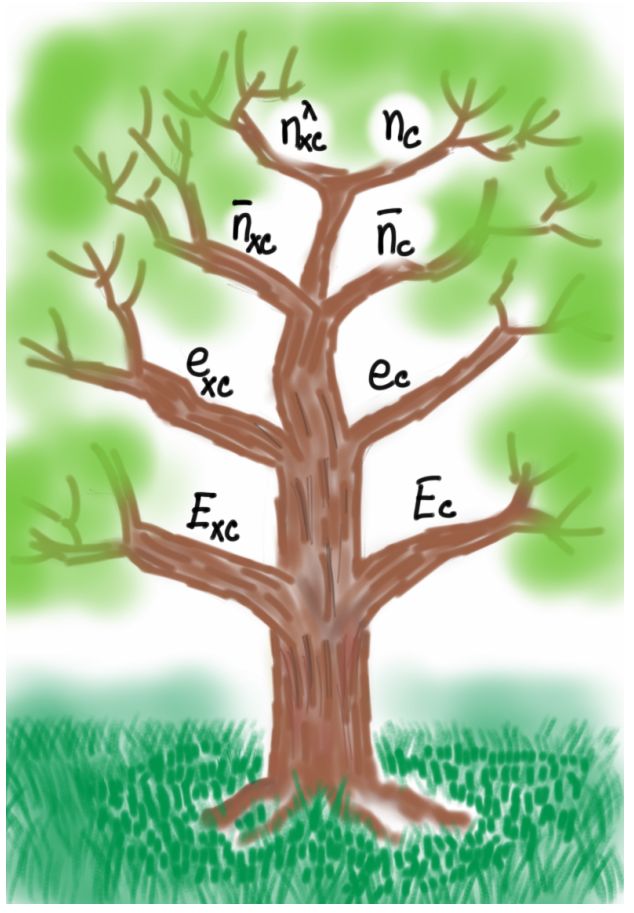

FIG. 3: The exchange-correlation tree (details in text)

asymptotics is to employ the idea of range separation [78, 79, 181-184], possibly combining it with local hybrid functionals. Range-separated hybrids, existing nowadays in various flavors, have been successfully applied to a variety of materials and chemical environments $[2,80-82,131,132,185-192]$. Yet, with the advantages of RSHs come also the challenges, particularly determining the range-separation parameter, which can be difficult e.g. in heterogeneous systems [193].

The results of this work suggest an additional possible route: instead on focussing on modelling $e_{\mathrm{xc}}[n](\mathbf{r})$, it is suggested here to invest in constructing approximations for the $\lambda$-averaged xc hole function, $\bar{n}_{\mathrm{xc}}\left(\mathbf{r}^{\prime \prime} \mid \mathbf{r}^{\prime}\right)$, satisfying the properties known for the hole and for its functional

\section{Appendix: OEP formalism for a complex functional}

The purpose of this appendix is to show how the optimized effective potential (OEP) formalism [90] can be generalized for the case of a complex functional. Usually, the OEP formalism serves in DFT to obtain the xc potential $v_{\mathrm{xc}}[n](\mathbf{r})$ from the xc energy functional $E_{\mathrm{xc}}[n]$, in cases where the xc energy functional is not explicitly density-dependent, but is rather explicitly orbitaldependent.

The xc energy is surely a real quantity, and standard OEP derivations that are found in the literature assume that. In Sec. IV we encounter a situation where the OEP derivative, $\bar{\eta}_{\mathrm{xc}}\left(\mathbf{r} ; \mathbf{r}^{\prime \prime} \mid \mathbf{r}^{\prime}\right)$. Alternatively, one can decide to use the exact exchange and search for a compatible correlation. In such a case, $n_{\mathrm{x}, \sigma}^{\text {exact }}\left(\mathbf{r}^{\prime \prime} \mid \mathbf{r}^{\prime}\right)$ and $\eta_{\mathrm{x}, \sigma}^{\text {exact }}\left(\mathbf{r} ; \mathbf{r}^{\prime \prime} \mid \mathbf{r}^{\prime}\right)$ are known (Eqs. (25) and (39)) and one searches for the correlation hole, i.e., for $\bar{n}_{\mathrm{c}}\left(\mathbf{r}^{\prime \prime} \mid \mathbf{r}^{\prime}\right)$ and $\bar{\eta}_{\mathrm{c}}\left(\mathbf{r} ; \mathbf{r}^{\prime \prime} \mid \mathbf{r}^{\prime}\right)$. Once the xc hole is approximated, obtaining the corresponding energy density per particle and the xc potential is straightforward (see Eq. (12) and (17)). This may open the door for approximations with correct asymptotics, both for $v_{\mathrm{xc}}[n](\mathbf{r})$ and $e_{\mathrm{xc}}[n](\mathbf{r})$.

The suggestion above can be further extended, going beyond the question of correct asymptotic behavior discussed here, to formulate a strategy for design of new xc approximations in general, following the concept of satisfying constraints. The idea is graphically presented in Fig. 3. This is a suggestion for classification of various xc functionals in rungs (or levels), as in "Jacob's ladder". Figure 3 shows a drawing of a tree with branches on four different levels. On each level a different DFT quantity is meant to be approximated. Climbing the tree, one progresses from more simple and intuitive concepts, such as [focusing on the left part for the moment] the xc energy, $E_{\mathrm{xc}}$ (level 1), or the xc energy density per particle, $e_{\mathrm{xc}}(\mathbf{r})$ (level 2) to the more abstract concepts of the $\lambda$-averaged xc hole, $\bar{n}_{\mathrm{xc}}\left(\mathbf{r}^{\prime} \mid \mathbf{r}\right)$ (level 3 ) all the way to the top of the tree, with the $\lambda$-dependent hole, $n_{\mathrm{xc}}^{(\lambda)}\left(\mathbf{r}^{\prime} \mid \mathbf{r}\right)$. The number of coordinates each quantity has, as well as the difficulty to construct approximations also rises. Of course, descending is usually easier than ascending: once an approximation for a quantity is made, those below are immediately obtained, by integration. In addition, one can choose whether to climb the tree on the left, approximating exchange and correlation, or on the right, using the exact exchange and looking for a compatible correlation functional. The various known xc approximations can be located on one of the branches of this tree. It generally seems that the first two levels are rather congested, whereas the higher levels are only sparsely occupied. This is a call to keep climbing the exchange-correlation tree! The sweetest fruits are on the top (not shown in Fig. 3).

formalism has to be applied to a complex quantity, and therefore the OEP equations have to be generalized accordingly, which is done below. In our derivation we closely follow Ref. [90], Sec. II. In particular, our results are presented in the spin-dependent form and we consider here the explicit dependence on the KS orbitals, but not on other quantities, such as the KS energies $\left\{\varepsilon_{i \sigma}\right\}$, the occupation numbers, etc.

We therefore consider a complex quantity, $Q$, which is an explicit functional of the KS orbitals: $Q\left[\left\{\varphi_{i \sigma}(\mathbf{r})\right\}\right]$, and therefore an implicit functional of the spin-densities, $n_{\sigma}(\mathbf{r})$. The first derivative of $Q$ with respect to $n_{\sigma}(\mathbf{r})$ is 
expressed as

$$
\begin{aligned}
& w_{\sigma}(\mathbf{r}):=\frac{\delta Q}{\delta n_{\sigma}(\mathbf{r})}= \\
& =\sum_{\alpha=\uparrow, \downarrow} \sum_{i=1}^{N_{\alpha}} \int d^{3} x\left[\frac{\delta Q}{\delta \varphi_{i \alpha}(\mathbf{x})} \frac{\delta \varphi_{i \alpha}(\mathbf{x})}{\delta n_{\sigma}(\mathbf{r})}+\frac{\delta Q}{\delta \varphi_{i \alpha}^{*}(\mathbf{x})} \frac{\delta \varphi_{i \alpha}^{*}(\mathbf{x})}{\delta n_{\sigma}(\mathbf{r})}\right]
\end{aligned}
$$

Unlike in a standard OEP derivation, here we write down explicitly both the derivative of $Q$ with respect to $\varphi_{i \alpha}(\mathbf{x})$ and with respect to $\varphi_{i \alpha}^{*}(\mathbf{x})$. If $Q$ were real, $\delta Q / \delta \varphi_{i \alpha}^{*}(\mathbf{x})$ would equal $\delta Q^{*} / \delta \varphi_{i \alpha}^{*}(\mathbf{x})$, which in turn would equal $\left(\delta Q / \delta \varphi_{i \alpha}(\mathbf{x})\right)^{*}$, and the second term of the integrand in Eq. (A.1) would then be simply the complex conjugate (c.c.) of the first term. However, when $Q$ is complex, both terms have to be explicitly considered.

The next few steps of the OEP derivation remain unaltered by the fact that $Q$ is complex: (i) the unknown derivative $\delta \varphi_{i \alpha}(\mathbf{x}) / \delta n_{\sigma}(\mathbf{r})$ is expressed using the KS potential as

$$
\frac{\delta \varphi_{i \alpha}(\mathbf{x})}{\delta n_{\sigma}(\mathbf{r})}=\sum_{\beta=\uparrow, \downarrow} \int d^{3} y \frac{\delta \varphi_{i \alpha}(\mathbf{x})}{\delta v_{\mathrm{KS}, \beta}(\mathbf{y})} \frac{\delta v_{\mathrm{KS}, \beta}(\mathbf{y})}{\delta n_{\sigma}(\mathbf{r})}
$$

and similarly for the derivative of $\varphi_{i \alpha}^{*}(\mathbf{x})$. (ii) The derivative of the KS orbital with respect to the KS potential can be derived from first-order perturbation theory

$$
\frac{\delta \varphi_{i \alpha}(\mathbf{x})}{\delta v_{\mathrm{KS}, \beta}(\mathbf{y})}=\delta_{\alpha \beta} \sum_{\substack{j=1 \\ j \neq i}}^{\infty} \frac{\varphi_{j \alpha}(\mathbf{x}) \varphi_{j \alpha}^{*}(\mathbf{y})}{\varepsilon_{i \alpha}-\varepsilon_{j \alpha}} \varphi_{i \alpha}(\mathbf{y})
$$

Again, the expression for the derivative of $\varphi_{i \alpha}^{*}(\mathbf{x})$ is very similar. (iii) The KS response function is given by

$$
\begin{aligned}
& \chi_{\sigma \beta}^{\mathrm{KS}}(\mathbf{r}, \mathbf{z})=\frac{\delta n_{\sigma}(\mathbf{r})}{\delta v_{\mathrm{KS}, \beta}(\mathbf{z})}= \\
& =\delta_{\sigma \beta} \sum_{i=1}^{N_{\sigma}} \sum_{\substack{j=1 \\
j \neq i}}^{\infty} \frac{\varphi_{i \sigma}^{*}(\mathbf{r}) \varphi_{j \sigma}^{*}(\mathbf{z}) \varphi_{i \sigma}(\mathbf{z}) \varphi_{j \sigma}(\mathbf{r})}{\varepsilon_{i \sigma}-\varepsilon_{j \sigma}}+c . c .
\end{aligned}
$$

Using the KS Green function

$$
G_{i \sigma}^{\mathrm{KS}}(\mathbf{x}, \mathbf{y})=\sum_{\substack{j=1 \\ j \neq i}}^{\infty} \frac{\varphi_{j \sigma}(\mathbf{x}) \varphi_{j \sigma}^{*}(\mathbf{y})}{\varepsilon_{i \sigma}-\varepsilon_{j \sigma}},
$$

we can express the results of Eqs. (A.3) and (A.4) as

$$
\begin{aligned}
\frac{\delta \varphi_{i \alpha}(\mathbf{x})}{\delta v_{\mathrm{KS}, \beta}(\mathbf{y})} & =\delta_{\alpha \beta} \varphi_{i \alpha}(\mathbf{y}) G_{i \alpha}^{\mathrm{KS}}(\mathbf{x}, \mathbf{y}) \\
\frac{\delta \varphi_{i \alpha}^{*}(\mathbf{x})}{\delta v_{\mathrm{KS}, \beta}(\mathbf{y})} & =\delta_{\alpha \beta} \varphi_{i \alpha}^{*}(\mathbf{y})\left[G_{i \alpha}^{\mathrm{KS}}(\mathbf{x}, \mathbf{y})\right]^{*}
\end{aligned}
$$

and

$$
\chi_{\sigma \beta}^{\mathrm{KS}}(\mathbf{r}, \mathbf{z})=\delta_{\sigma \beta} \sum_{i=1}^{N_{\sigma}} \varphi_{i \sigma}^{*}(\mathbf{r}) \varphi_{i \sigma}(\mathbf{z}) G_{i \sigma}^{\mathrm{KS}}(\mathbf{r}, \mathbf{z})+c . c .
$$

The fact that results (i)-(iii) remain unchanged for a complex $Q$ is not surprising: they have nothing to do with the functional $Q$, whose derivative we are looking for.

Next, we denote the orbital-specific derivatives

$$
u_{i \sigma}(\mathbf{r}):=\frac{1}{\varphi_{i \sigma}^{*}(\mathbf{r})} \frac{\delta Q}{\delta \varphi_{i \sigma}(\mathbf{r})}, \quad \widetilde{u}_{i \sigma}(\mathbf{r}):=\frac{1}{\varphi_{i \sigma}(\mathbf{r})} \frac{\delta Q}{\delta \varphi_{i \sigma}^{*}(\mathbf{r})} .
$$

For the particular case of a real $Q$, we have $\widetilde{u}_{i \sigma}(\mathbf{r})=$ $u_{i \sigma}^{*}(\mathbf{r})$; not so for a complex $Q$. We now substitute Eqs. (A.2), (A.6), (A.7) and (A.9) into Eq. (A.1) to obtain:

$$
\begin{aligned}
w_{\sigma}(\mathbf{r})= & \sum_{\alpha} \sum_{i=1}^{N_{\alpha}} \iint d^{3} x d^{3} y \frac{\delta v_{\mathrm{KS}, \alpha}(\mathbf{y})}{\delta n_{\sigma}(\mathbf{r})} \\
& \cdot\left[u_{i \alpha}(\mathbf{x}) \varphi_{i \alpha}^{*}(\mathbf{x}) \varphi_{i \alpha}(\mathbf{y}) G_{i \alpha}^{\mathrm{KS}}(\mathbf{x}, \mathbf{y})+\right. \\
& \left.+\widetilde{u}_{i \alpha}(\mathbf{x}) \varphi_{i \alpha}(\mathbf{x}) \varphi_{i \alpha}^{*}(\mathbf{y})\left[G_{i \alpha}^{\mathrm{KS}}(\mathbf{x}, \mathbf{y})\right]^{*}\right]
\end{aligned}
$$

We multiply both sides of this equation by $\chi_{\sigma \beta}^{\mathrm{KS}}(\mathbf{r}, \mathbf{z})$, integrate over $\mathbf{r}$ and sum over $\beta$. Then, on the left-hand-side we exchange the variables $\mathbf{r} \mapsto \mathbf{x}$, and only then on both sides $\mathbf{z} \mapsto \mathbf{r}$ to obtain the celebrated OEP equation

$$
\begin{aligned}
& \sum_{i=1}^{N_{\sigma}} \int d^{3} x\left[\varphi_{i \sigma}^{*}(\mathbf{x})\left(w_{\sigma}(\mathbf{x})-u_{i \sigma}(\mathbf{x})\right) \varphi_{i \sigma}(\mathbf{r}) G_{i \sigma}^{\mathrm{KS}}(\mathbf{x}, \mathbf{r})+\right. \\
& \left.+\varphi_{i \sigma}(\mathbf{x})\left(w_{\sigma}(\mathbf{x})-\widetilde{u}_{i \sigma}(\mathbf{x})\right) \varphi_{i \sigma}^{*}(\mathbf{r})\left[G_{i \sigma}^{\mathrm{KS}}(\mathbf{x}, \mathbf{r})\right]^{*}\right]=0
\end{aligned}
$$

generalized for a complex $Q$. Notably, in the case of a real $Q$, where $\widetilde{u}_{i \sigma}(\mathbf{r})=u_{i \sigma}^{*}(\mathbf{r})$, the second term of Eq. (A.11) becomes the complex conjugate of the first term, and the OEP equation reduces to its known form (Eq. (24) of Ref. [90]).

We continue the derivation further, aiming to resolve Eq. (A.11) with respect to $w_{\sigma}(\mathbf{r})$, by introducing the socalled orbital shifts. The first orbital shift

$$
\psi_{i \sigma}^{*}(\mathbf{r})=\int d^{3} x \varphi_{i \sigma}^{*}(\mathbf{x})\left(w_{\sigma}(\mathbf{x})-u_{i \sigma}(\mathbf{x})\right) G_{i \sigma}^{\mathrm{KS}}(\mathbf{x}, \mathbf{r})
$$

(A.12)

is the familiar one, whereas the second, complimentary orbital shift reads

$$
\widetilde{\psi}_{i \sigma}(\mathbf{r})=\int d^{3} x \varphi_{i \sigma}(\mathbf{x})\left(w_{\sigma}(\mathbf{x})-\widetilde{u}_{i \sigma}(\mathbf{x})\right)\left[G_{i \sigma}^{\mathrm{KS}}(\mathbf{x}, \mathbf{r})\right]^{*} .
$$

These definitions allow us to express the generalized OEP equation, Eq. (A.11), as

$$
\sum_{i=1}^{N_{\sigma}} \varphi_{i \sigma}(\mathbf{r}) \psi_{i \sigma}^{*}(\mathbf{r})+\varphi_{i \sigma}^{*}(\mathbf{r}) \widetilde{\psi}_{i \sigma}(\mathbf{r})=0
$$

Again, for a real $Q, w_{\sigma}(\mathbf{r})$ is real, $\widetilde{\psi}_{i \sigma}(\mathbf{r})=\psi_{i \sigma}(\mathbf{r})$ and therefore Eq. (A.14) reduces to its usual form. 
The KS Green function satisfies the following inhomogeneous Schrödinger-like equation:

$$
\begin{aligned}
\left(-\frac{1}{2} \nabla^{2}+v_{\mathrm{KS}, \sigma}\right. & \left.(\mathbf{r})-\varepsilon_{i \sigma}\right) G_{i \sigma}^{\mathrm{KS}}(\mathbf{x}, \mathbf{r})= \\
= & -\left[\delta(\mathbf{x}-\mathbf{r})-\varphi_{i \sigma}(\mathbf{x}) \varphi_{i \sigma}^{*}(\mathbf{r})\right] .
\end{aligned}
$$

As a result, one deduces the following equations for the orbital shifts:

$$
\begin{aligned}
& \left(-\frac{1}{2} \nabla^{2}+v_{\mathrm{KS}, \sigma}(\mathbf{r})-\varepsilon_{i \sigma}\right) \psi_{i \sigma}^{*}(\mathbf{r})= \\
& \quad=-\left[\left(w_{\sigma}(\mathbf{r})-u_{i \sigma}(\mathbf{r})\right)-\left(\bar{w}_{i \sigma}-\bar{u}_{i \sigma}\right)\right] \varphi_{i \sigma}^{*}(\mathbf{r}) \\
& \left(-\frac{1}{2} \nabla^{2}+v_{\mathrm{KS}, \sigma}(\mathbf{r})-\varepsilon_{i \sigma}\right) \widetilde{\psi}_{i \sigma}(\mathbf{r})= \\
& \quad=-\left[\left(w_{\sigma}(\mathbf{r})-\widetilde{u}_{i \sigma}(\mathbf{r})\right)-\left(\bar{w}_{i \sigma}-\overline{\widetilde{u}}_{i \sigma}\right)\right] \varphi_{i \sigma}(\mathbf{r})
\end{aligned}
$$

where the bar above a quantity means its average with respect to the $i^{\text {th }}$ KS orbital, e.g., $\bar{w}_{i \sigma}=\int d^{3} r \varphi_{i \sigma}^{*}(\mathbf{r}) w_{\sigma}(\mathbf{r}) \varphi_{i \sigma}(\mathbf{r}) . \quad$ Equations (A.16) and (A.17) can be resolved with respect to $v_{\mathrm{KS}, \sigma}(\mathbf{r}) \psi_{i \sigma}^{*}(\mathbf{r})$ and $v_{\mathrm{KS}, \sigma}(\mathbf{r}) \widetilde{\psi}_{i \sigma}(\mathbf{r})$, respectively, and substituted into Eq. (A.14) to finally get an expression for $w_{\sigma}(\mathbf{r})$ :

$$
\begin{aligned}
w_{\sigma}(\mathbf{r})= & \frac{1}{n_{\sigma}(\mathbf{r})}\left\{\sum_{i=1}^{N_{\sigma}}\left[U_{i \sigma}(\mathbf{r})+\left(\bar{w}_{i \sigma}-\bar{U}_{i \sigma}\right)\right]\left|\varphi_{i \sigma}(\mathbf{r})\right|^{2}+\right. \\
+ & \frac{1}{2}\left[\varphi_{i \sigma}(\mathbf{r})\left(\frac{1}{2} \nabla^{2}+\varepsilon_{i \sigma}\right) \psi_{i \sigma}^{*}(\mathbf{r})+\right. \\
& \left.\left.+\varphi_{i \sigma}^{*}(\mathbf{r})\left(\frac{1}{2} \nabla^{2}+\varepsilon_{i \sigma}\right) \widetilde{\psi}_{i \sigma}(\mathbf{r})\right]\right\}(\mathrm{A} .18)
\end{aligned}
$$

Here

$$
U_{i \sigma}(\mathbf{r})=\frac{1}{2}\left(u_{i \sigma}(\mathbf{r})+\widetilde{u}_{i \sigma}(\mathbf{r})\right)
$$

which can be formally viewed as the average of the two orbital-specific derivatives of $Q$, defined in Eq. (A.9). Finally, using the same algebraic techniques as in the standard OEP derivation, Eq. (A.18) can be further simplified to

$$
\begin{aligned}
w_{\sigma}(\mathbf{r}) & =\frac{1}{n_{\sigma}(\mathbf{r})}\left\{\sum_{i=1}^{N_{\sigma}}\left[U_{i \sigma}(\mathbf{r})+\left(\bar{w}_{i \sigma}-\bar{U}_{i \sigma}\right)\right]\left|\varphi_{i \sigma}(\mathbf{r})\right|^{2}-\right. \\
& \left.-\frac{1}{2} \nabla \cdot\left(\psi_{i \sigma}^{*}(\mathbf{r}) \nabla \varphi_{i \sigma}(\mathbf{r})+\widetilde{\psi}_{i \sigma}(\mathbf{r}) \nabla \varphi_{i \sigma}^{*}(\mathbf{r})\right)\right\} .
\end{aligned}
$$

Equation (A.20) provides an expression for $w_{\sigma}(\mathbf{r})-$ the functional derivative of a complex, explicit orbitaldependent functional $Q$, with respect to the density, in terms of the generalized orbital specific derivatives $U_{i \sigma}(\mathbf{r})$ and the orbital shifts $\psi_{i \sigma}^{*}(\mathbf{r})$ and $\widetilde{\psi}_{i \sigma}(\mathbf{r})$. Equation (A.20) serves as the the generalization of the usual $w_{\sigma}$-resolved OEP equation (cf. Eq. (35) of Ref. [90]) for the case of a complex $Q$. The derivation presented here allows us to address the derivative of the $\mathrm{KS}$ one-electron reduced density matrix with respect to the spin density, which is discussed in the main text.

\section{Acknowledgments}

I would like to express my gratitude to Prof. Stephan Kümmel for his advice and to Dr. Axel Schild for fruitful discussions of the EEF approach and his useful comments on the manuscript.
[1] C. DiValentin, S. Botti, and M. Cococcioni, eds., First Principle Approaches to Spectroscopic Properties of Complex Materials (Springer, 2014), vol. 347 of Topics in Current Chemistry.

[2] L. Kronik and J. B. Neaton, Annu. Rev. Phys. Chem. 67, 587 (2016).

[3] S. Kümmel, Adv. Energy Mater. 7, 1700440 (2017).

[4] G. J. Hedley, A. Ruseckas, and I. D. W. Samuel, Chem. Rev. 117, 796 (2017).

[5] C. Curutchet and B. Mennucci, Chem. Rev. 117, 294 (2017).

[6] A. R. Oganov, ed., Methods and applications of crystal structure prediction, vol. 211 of Faraday Discuss. (2018).

[7] R. J. Maurer, C. Freysoldt, A. M. Reilly, J. G. Brandenburg, O. T. Hofmann, T. Björkman, S. Lebègue, and A. Tkatchenko, Annu. Rev. Mater. Res. 49, 3.1 (2019).

[8] R.M. Dreizler and E.K.U. Gross, Density Functional Theory (Springer Verlag, 1990).
[9] R. G. Parr and W. Yang, Density-Functional Theory of Atoms and Molecules (Oxford University Press, 1989).

[10] C. Fiolhais, F. Nogueira, and M. A. Marques, eds., A Primer in Density Functional Theory (Springer, 2003).

[11] E. Engel and R. Dreizler, Density Functional Theory: An Advanced Course (Springer, 2011).

[12] E. Kaxiras, Atomic and Electronic Structure of Solids (Cambridge University Press, 2003).

[13] R. Martin, Electronic Structure (Cambridge Unviersity Press, 2004).

[14] C. Cramer, Essentials Of Computational Chemistry: Theories And Models (Wiley, 2004).

[15] J. Kohanoff, Electronic Structure Calculations For Solids And Molecules: Theory And Computational Methods (Cambridge University Press, 2006).

[16] D. Sholl and J. Steckel, Density Functional Theory: A Practical Introduction (Wiley, 2011).

[17] F. Giustino, Materials modelling using density func- 
tional theory: properties and predictions (Oxford University Press, 2014).

[18] K. Burke, J. Chem. Phys. 136, 150901 (2012).

[19] A. D. Becke, J. Chem. Phys. 140, 18A301 (2014).

[20] R. O. Jones, Rev. Mod. Phys. 87, 897 (2015).

[21] P. Hohenberg and W. Kohn, Phys. Rev. 136, B864 (1964).

[22] M. Levy, Proc. Nat. Acad. Sci. 76, 6062 (1979).

[23] M. Levy, Phys. Rev. A 26, 1200 (1982).

[24] W. Kohn and L. J. Sham, Phys. Rev. 140, A1133 (1965).

[25] J. P. Perdew and K. Schmidt, in Density Functional Theory and Its Application to Materials, edited by V. Van Doren, C. Van Alsenoy, and P. Geerlings (AIP, 2001).

[26] J. P. Perdew, A. Ruzsinszky, L. A. Constantin, J. Sun, and G. I. Csonka, J. Chem. Theory Comp. 5, 902 (2009).

[27] N. Mardirossian and M. Head-Gordon, Mol. Phys. 115, 2315 (2017).

[28] V. Staroverov, G. Scuseria, J. Tao, and J. P. Perdew, Phys. Rev. B 69, 075102 (2004).

[29] P. Haas, F. Tran, and P. Blaha, Phys. Rev. B 79, 085104 (2009).

[30] P. Haas, F. Tran, P. Blaha, and K. Schwarz, Phys. Rev. B 83, 205117 (2011).

[31] F. Tran, P. Blaha, M. Betzinger, and S. Blügel, Phys. Rev. B 91, 165121 (2015).

[32] F. Tran, J. Stezl, and P. Blaha, J. Chem. Phys. 144, 204120 (2016).

[33] E. Kraisler, T. Schmidt, S. Kümmel, and L. Kronik, J. Chem. Phys. 143, 104105 (2015).

[34] P. Borlido, T. Aull, A. W. Huran, F. Tran, M. A. L. Marques, and S. Botti, J. Chem. Theory Comput. (2019).

[35] J. P. Perdew, J. Chem. Phys. 123, 062201 (2005).

[36] J. P. Perdew, A. Ruzsinszky, J. Sun, and K. Burke, J. Chem. Phys. 140, 18A533 (2014).

[37] J. P. Perdew, J. Sun, R. M. Martin, and B. Delley, Int. J. Quantum Chem. 116, 847 (2016).

[38] J. P. Perdew, K. Burke, and M. Ernzerhof, Phys. Rev. Lett. 77, 3865 (1996).

[39] J. Sun, A. Ruzsinszky, and J. P. Perdew, Phys. Rev. Lett. 115, 036402 (2015).

[40] J. Sun, R. C. Remsing, Y. Zhang, Z. Sun, A. Ruzsinszky, H. Peng, Z. Yang, A. Paul, U. Waghmare, X. Wu, et al., Nature Chemistry 8, 831 (2016).

[41] C. Filippi, X. Gonze, and C. Umrigar, in Recent Developments and Applications of Modern Density Functional Theory, edited by J. Seminario (Elsevier Science, 1996), vol. 4 of Theoretical and Computational Chemistry, pp. 295-326.

[42] J. Katriel and E. R. Davidson, PNAS 77, 4403 (1980).

[43] J. P. Perdew and A. Zunger, Phys. Rev. B 23, 5048 (1981).

[44] E. Lieb and S. Oxford, Int. J. Quantum Chem. 19, 427 (1981).

[45] J. P. Perdew, R. G. Parr, M. Levy, and J. L. Balduz, Phys. Rev. Lett. 49, 1691 (1982).

[46] J. P. Perdew and M. Levy, Phys. Rev. Lett. 51, 1884 (1983).

[47] L. J. Sham and M. Schlüter, Phys. Rev. Lett. 51, 1888 (1983).

[48] M. Levy, J. P. Perdew, and V. Sahni, Phys. Rev. A 30, 2745 (1984).
[49] M. Levy, Phys. Rev. A 32, 2010 (1985)

[50] C. O. Almbladh and U. von Barth, Phys. Rev. B 31, 3231 (1985).

[51] C. O. Almbladh and U. von Barth, in Density Functional Methods in Physics, edited by R. M. Dreizler and J. da Providência (Plenum Press, 1985), vol. 123 of NATO ASI Series, pp. 209-231.

[52] J. P. Perdew, in Density Functional Methods in Physics, edited by R. M. Dreizler and J. da Providência (Plenum Press, 1985), vol. 123 of NATO ASI Series, pp. 265-308.

[53] W. Kohn, Phys. Rev. B 33, 4331 (1986).

[54] M. Levy and H. Ou-Yang, Phys. Rev. A 42, 651 (1990).

[55] H. Ou-Yang and M. Levy, Phys. Rev. A 42, 155 (1990).

[56] J. P. Perdew, Adv. Quantum Chem. 21, 113 (1990).

[57] M. Levy, Phys. Rev. A 43, 4637 (1991).

[58] A. Görling and M. Levy, Phys. Rev. A 45, 1509 (1992).

[59] M. Lein and S. Kümmel, Phys. Rev. Lett. 94, 143003 (2005).

[60] W. Kohn, Phys. Rev. Lett. 76, 3168 (1996).

[61] E. Prodan and W. Kohn, PNAS 102, 11635 (2005).

[62] M. Mundt and S. Kümmel, Phys. Rev. Lett. 95, 203004 (2005).

[63] P. Mori-Sánchez, A. J. Cohen, and W. Yang, J. Chem. Phys. 125, 201102 (2006).

[64] J. Perdew, V. Staroverov, J. Tao, and G. Scuseria, Phys. Rev. A 78, 052513 (2008).

[65] A. J. Cohen, P. Mori-Sánchez, and W. Yang, Science 321, 792 (2008).

[66] P. Mori-Sánchez, A. J. Cohen, and W. Yang, Phys. Rev. Lett. 100, 146401 (2008).

[67] A. J. Cohen, P. Mori-Sánchez, and W. Yang, Chem. Rev. 112, 289 (2012).

[68] W. Yang, A. J. Cohen, and P. Mori-Sánchez, J. Chem. Phys. 136, 204111 (2012).

[69] M. Hellgren and E. K. U. Gross, Phys. Rev. A 85, 022514 (2012).

[70] M. J. P. Hodgson, J. D. Ramsden, and R. W. Godby, Phys. Rev. B 93, 155146 (2016).

[71] T. Schmidt, E. Kraisler, L. Kronik, and S. Kümmel, Phys. Chem. Chem. Phys. 16, 14357 (2014).

[72] M. J. P. Hodgson, E. Kraisler, A. Schild, and E. K. U. Gross, J. Phys. Chem. Lett. 8, 5974 (2017).

[73] J. P. Perdew and M. Levy, Phys. Rev. B 56, 16021 (1997).

[74] M. K. Harbola, Phys. Rev. B 60, 4545 (1999).

[75] D. J. Tozer and N. C. Handy, J. Chem. Phys. 109, 10180 (1998).

[76] D. J. Tozer, R. D. Amos, N. C. Handy, B. O. Ross, and L. Serrano-Andres, Mol. Phys. 97, 859 (1999).

[77] M. E. Casida and D. R. Salahub, J. Chem. Phys. 113, 8918 (2000).

[78] R. Baer, E. Livshits, and U. Salzner, Annu. Rev. Phys. Chem. 61, 85 (2010).

[79] L. Kronik, T. Stein, S. Refaely-Abramson, and R. Baer, J. Chem. Theory Comp. 8, 1515 (2012).

[80] L. Kronik and S. Kümmel, in Topics of Current Chemistry: First Principles Approaches to Spectroscopic Properties of Complex Materials, edited by C. di Valentin, S. Botti, and M. Coccoccioni (Springer, Berlin, 2014), vol. 347, pp. 137-192.

[81] L. Kronik and S. Kmmel, Adv. Mater. 30, 1706560 (2018).

[82] D. Wing, J. B. Haber, R. Noff, B. Barker, D. A. Egger, A. Ramasubramaniam, S. G. Louie, J. B. Neaton, and 
L. Kronik, Phys. Rev. Materials 3, 064603 (2019).

[83] T. Gould and J. F. Dobson, J. Chem. Phys. 138, 014103 (2013).

[84] T. Gould and J. Toulouse, Phys. Rev. A 90, 050502 (R) (2014).

[85] E. Kraisler and L. Kronik, Phys. Rev. Lett. 110, 126403 (2013).

[86] T. Gould, S. Pittalis, J. Toulouse, E. Kraisler, and L. Kronik, Asymptotic behavior of the Hartree-exchange and correlation potentials for fractional electron numbers in atoms, Phys. Chem. Chem. Phys. (submitted). ChemRxiv preprint at https://doi.org/10.26434/chemrxiv.8340857.v1.

[87] F. Della Sala and A. Görling, J. Chem. Phys. 116, 5374 (2002).

[88] S. Kümmel and J. P. Perdew, Phys. Rev. B 68, 035103 (2003).

[89] D. P. Joubert, Phys. Rev. A 76, 012501 (2007).

[90] S. Kümmel and L. Kronik, Rev. Mod. Phys. 80, 3 (2008).

[91] P. Gori-Giorgi, T. Gál, and E. J. Baerends, Mol. Phys. 114, 1086 (2016).

[92] T. Aschebrock, R. Armiento, and S. Kümmel, Phys. Rev. B 95, 245118 (2017).

[93] P. Gori-Giorgi and E. J. Baerends, Eur. Phys. J. B 91, 160 (2018).

[94] A. V. Arbuznikov and M. Kaupp, J. Chem. Phys. 141, 204101 (2014).

[95] T. M. Maier, M. Haasler, A. V. Arbuznikov, and M. Kaupp, Phys. Chem. Chem. Phys. 18, 21133 (2016).

[96] T. M. Maier, A. V. Arbuznikov, and M. Kaupp, WIREs: Comput. Mol. Sci. 9, e1378 (2019).

[97] O. Gunnarsson and B. I. Lundqvist, Phys. Rev. B 13, 4274 (1976).

[98] O. V. Gritsenko and E. J. Baerends, Int. J. Quantum Chem. 101, 5383 (1997).

[99] A. D. Becke, J. Chem. Phys. 138, 074109 (2013).

[100] J. Harris and R. O. Jones, J. Phys. F: Met. Phys. 4, 1170 (1974).

[101] M. Ernzerhof, J. P. Perdew, and K. Burke, Int. J. Quantum Chem. 64, 285 (1997).

[102] R. P. Feynman, Phys. Rev. 56, 340 (1939).

[103] L. D. Landau and E. M. Lifshitz, Quantum Mechanics (Non-Relativistic Theory), vol. 3 of Course of Theoretical Physics (Pergamon, 1991), 3rd ed.

[104] A. Schild and E. K. U. Gross, Phys. Rev. Lett. 118, 163202 (2017).

[105] A. Abedi, N. T. Maitra, and E. K. Gross, Phys. Rev. Lett. 105, 123002 (2010).

[106] A. Abedi, F. Agostini, Y. Suzuki, and E. K. U. Gross, Phys. Rev. Lett. 110, 263001 (2013).

[107] N. I. Gidopoulos and E. K. U. Gross, Trans. R. Soc. A 372, 20130059 (2014).

[108] N. H. March, Phys. Lett. 113A, 476 (1986).

[109] M. Levy and H. Ou-Yang, Phys. Rev. A 38, 625 (1988).

[110] Y. A. Wang and E. A. Carter, in Theoretical methods in condensed phase chemistry, edited by S. D. Schwartz (Springer, Berlin, 2002), pp. 117-184.

[111] V. Lignères and E. A. Carter, in Handbook of Materials Modeling, edited by S. Yip (Springer Netherlands, 2005), chap. 1.8, pp. 137-148.

[112] J.-D. Chai, V. L. Lignères, G. Ho, E. A. Carter, and J. D. Weeks, Chem. Phys. Lett. 473, 263 (2009).

[113] V. V. Karasiev and S. B. Trickey, Comput. Phys. Com- mun. 183, 2519 (2012).

[114] H. Levämäki, Á. Nagy, K. Kokko, and L. Vitos, Phys. Rev. A 92, 062502 (2015).

[115] K. Finzel, Comput. Theor. Chem. 1144, 50 (2018).

[116] K. Finzel, J. Chem. Phys. 151, 024109 (2019).

[117] J. B. Krieger, Y. Li, and G. J. Iafrate, Phys. Rev. A 46, 5453 (1992).

[118] T. Kreibich, S. Kurth, T. Grabo, and E. K. U. Gross, Adv. Quantum Chem. 33, 31 (1998).

[119] S. H. Vosko, L. Wilk, and M. Nusair, Can. J. Phys. 58, 1200 (1980).

[120] J. P. Perdew and Y. Wang, Phys. Rev. B 45, 13244 (1992).

[121] J. Tao, J. P. Perdew, V. Staroverov, and G. Scuseria, Phys. Rev. Lett. 91, 146401 (2003).

[122] J. P. Perdew, M. Ernzerhof, and K. Burke, J. Chem. Phys. 105, 9982 (1996).

[123] M. Ernzerhof and G. E. Scuseria, J. Chem. Phys. 110, 5029 (1999).

[124] C. Adamo and V. Barone, J. Chem. Phys. 110, 6158 (1999).

[125] A. D. Becke, J. Chem. Phys. 98, 5648 (1993).

[126] P. J. Stephens, F. J. Devlin, C. F. Chabalowski, and M. J. Frisch, J. Phys. Chem. 98, 11623 (1994).

[127] F. G. Cruz, K.-C. Lam, and K. Burke, J. Phys. Chem. A 102, 4911 (1998).

[128] J. Jaramillo, G. E. Scuseria, and M. Ernzerhof, J. Chem. Phys. 118, 1068 (2003).

[129] T. Schmidt, E. Kraisler, A. Makmal, L. Kronik, and S. Kümmel, J. Chem. Phys. 140, 18A510 (2014).

[130] P. de Silva and C. Corminboeuf, J. Chem. Phys. 142, 074112 (2015).

[131] S. Refaely-Abramson, S. Sharifzadeh, M. Jain, R. Baer, J. B. Neaton, and L. Kronik, Phys. Rev. B 88, 081204 (2013).

[132] S. Refaely-Abramson, M. Jain, S. Sharifzadeh, J. B. Neaton, and L. Kronik, Phys. Rev. B 92, 081204 (2015).

[133] D. C. Langreth and J. P. Perdew, Phys. Rev. B 21, 5469 (1980).

[134] A. D. Becke, Phys. Rev. A 38, 3098 (1988).

[135] R. Armiento and S. Kümmel, Phys. Rev. Lett. 111, 036402 (2013).

[136] V. Atalla, I. Y. Zhang, O. T. Hofmann, X. Ren, P. Rinke, and M. Scheffler, Phys. Rev. B 94, 035140 (2016).

[137] S. Kotochigova, Z. H. Levine, E. L. Shirley, M. D. Stiles, and C. W. Clark, Phys. Rev. A 55, 191 (1997).

[138] E. Kraisler, G. Makov, and I. Kelson, Phys. Rev. A 82, 042516 (2010).

[139] S. Kümmel, Adv. Atom. Mol. Opt. Phys. 64, 143 (2015).

[140] A. Svane and O. Gunnarsson, Phys. Rev. Lett. 65, 1148 (1990).

[141] W. M. Temmerman, Z. Szotek, and H. Winter, Phys. Rev. B 47, 11533 (1993).

[142] P. Strange, A. Svane, W. M. Temmerman, Z. Szotek, and H. Winter, Nature 399, 756 (1999).

[143] E. Engel and R. Schmid, Phys. Rev. Lett 103, 036404 (2009).

[144] X.-M. Tong and S.-I. Chu, Phys. Rev. A 55, 3406 (1997).

[145] S.-I. Chu, J. Chem. Phys. 123, 062207 (2005).

[146] C. A. Ullrich, P.-G. Reinhard, and E. Suraud, Phys. Rev. A 62, 053202 (2000). 
[147] D. A. Telnov, T. Heslar, and S.-I. Chu, Chem. Phys. 391, 88 (2011).

[148] T. Körzdörfer, M. Mundt, and S. Kümmel, Phys. Rev. Lett. 100, 133004 (2008).

[149] D. Hofmann, T. Körzdörfer, and S. Kümmel, Phys. Rev. Lett. 108, 146401 (2012).

[150] D. Hofmann and S. Kümmel, Phys. Rev. B 86, 201109 (2012).

[151] T. Schmidt and S. Kümmel, Phys. Rev. B 93, 165120 (2016).

[152] T. Bally and G. N. Sastry, J. Phys. Chem. A 101, 7923 (1997).

[153] A. Ruzsinszky, J. P. Perdew, and G. I. Csonka, J. Phys. Chem. A 109, 11006 (2005).

[154] A. Ruzsinszky, J. P. Perdew, G. I. Csonka, O. A. Vydrov, and G. E. Scuseria, J. Chem. Phys. 125, 194112 (2006).

[155] E. Livshits and R. Baer, J. Phys. Chem. A 112, 12789 (2008).

[156] A. D. Dwyer and D. J. Tozer, J. Chem. Phys. 135, 164110 (2011).

[157] J. Nafziger and A. Wasserman, J. Phys. Chem. A 118, 7623 (2014)

[158] J. Nafziger and A. Wasserman, J. Chem. Phys. 143, 234105 (2015).

[159] D. P. Chong, O. V. Gritsenko, and E. J. Baerends, J. Chem. Phys. 116, 1760 (2002).

[160] T. Körzdörfer, S. Kümmel, N. Marom, and L. Kronik, 79, 201205 (2009), ibid. 82, 129903(E) (2010).

[161] T. Körzdörfer and S. Kümmel, Phys. Rev. B 82, 155206 (2010).

[162] M. Dauth, T. Körzdörfer, S. Kümmel, J. Ziroff, M. Wiessner, A. Schöll, F. Reinert, M. Arita, and K. Shimada, Phys. Rev. Lett. 107, 193002 (2011).

[163] T. Schmidt and S. Kmmel, Computation 4 (2016).

[164] O. A. Vydrov, G. E. Scuseria, J. P. Perdew, A. Ruzsinszky, and G. I. Csonka, J. Chem. Phys. 124, 094108 (2006).

[165] T. Stein, J. Autschbach, N. Govind, L. Kronik, and R. Baer, J. Phys. Chem. Lett. 3, 3740 (2012).

[166] W. Yang, P. Mori-Sánchez, and A. J. Cohen, J. Chem. Phys. 139, 104114 (2013).

[167] P. Elliott, K. Burke, M. H. Cohen, and A. Wasserman, Phys. Rev. A 82, 024501 (2010).

[168] M. A. Mosquera and A. Wasserman, Phys. Rev. A 89, 052506 (2014).

[169] A. Benítez and C. R. Proetto, Phys. Rev. A 94, 052506 (2016).

[170] M. Dauth, F. Caruso, S. Kümmel, and P. Rinke, Phys. Rev. B 93, 121115(R) (2016).

[171] I. Dabo, A. Ferretti, and N. Marzari, Top. Curr. Chem. 347, 193 (2014).

[172] B. Senjean and E. Fromager, Phys. Rev. A 98, 022513 (2018).

[173] K. Deur and E. Fromager, J. Chem. Phys. 150, 094106 (2019).

[174] R. van Leeuwen and E. J. Baerends, Phys. Rev. A 49, 2421 (1994).

[175] A. D. Becke and E. R. Johnson, J. Chem. Phys. 124, 221101 (2006).

[176] F. Tran and P. Blaha, Phys. Rev. Lett. 102, 226401 (2009).

[177] W. Cencek and K. Szalewicz, J. Chem. Phys. 139, 024104 (2013).
[178] A. Karolewski, R. Armiento, and S. Kümmel, J. Chem. Theory Comp. 5, 712 (2009).

[179] A. P. Gaiduk, S. Chulkov, and V. N. Staroverov, J. Chem. Theory Comput. 5, 699 (2009).

[180] A. Karolewski, R. Armiento, and S. Kümmel, Phys. Rev. A 88, 052519 (2013).

[181] A. Savin, in Recent Advances in Density Functional Methods. Part I, edited by D. P. Chong (World Scientific: Singapore, 1995), p. 129.

[182] H. J. Savin, A.; Flad, Int. J. Quantum Chem. 56, 327 (1995).

[183] T. Leininger, H. Stoll, H.-J. Werner, and A. Savin, Chem. Phys. Lett. 275, 151 (1997).

[184] H. Iikura, T. Tsuneda, T. Yanai, and K. J. Hirao, Chem. Phys. 115, 3540 (2001).

[185] T. Stein, H. Eisenberg, L. Kronik, and R. Baer, Phys. Rev. Lett. 105, 266802 (2010).

[186] S. Refaely-Abramson, R. Baer, and L. Kronik, Phys. Rev. B 84, 075144 (2011).

[187] S. Refaely-Abramson, S. Sharifzadeh, N. Govind, J. Autschbach, J. B. Neaton, R. Baer, and L. Kronik, Phys. Rev. Lett. 109, 226405 (2012).

[188] E. Livshits and R. Baer, Phys. Chem. Chem. Phys. 9, 2932 (2007).

[189] D. A. Egger, S. Weissman, S. Refaely-Abramson, S. Sharifzadeh, M. Dauth, R. Baer, S. Kmmel, J. B. Neaton, E. Zojer, and L. Kronik, J. Chem. Theory Comput. 10, 1934 (2014).

[190] V. Vlček, G. Steinle-Neumann, L. Leppert, R. Armiento, and S. Kümmel, Phys. Rev. B 91, 035107 (2015).

[191] D. Neuhauser, E. Rabani, Y. Cytter, and R. Baer, J. Phys. Chem. A 120, 3071 (2016).

[192] J. H. Skone, M. Govoni, and G. Galli, Phys. Rev. B 93, 235106 (2016).

[193] A. Karolewski, L. Kronik, and S. Kümmel, J. Chem. Phys. 138, 204115 (2013).

[194] M. Born and R. Oppenheimer, Ann. Phys. Leipzig 84, 457 (1927).

[195] M. Born and K. Huang, Dynamical theory of crystal lattices (Oxford University Press, 1954).

[196] J. Jackson, Classical Electrodynamics (Wiley, 1962).

[197] By presenting all the aforementioned systems as manyelectron systems, we are making the Born-Oppenheimer approximation $[13,194,195]$ : we assume that the nuclei of the material are moving much slower than the electrons due to their large masses, and that the electrons always adapt themselves to the nuclear motion. This allows us to focus on the electronic part of the problem alone.

[198] The one-to-one mapping $n \leftrightarrow v_{\text {ext }}$ exists in case the ground state of the system is not degenerate; otherwise, only the mapping $n \mapsto v_{\text {ext }}$ holds. The latter is enough, however, to establish DFT (see, e.g., [8] for more details).

[199] Hartree atomic units are used throughout.

[200] For simplicity of presentation, here we use the spinindependent version of KS-DFT. For spin-DFT see, e.g., $[8-10]$

[201] In practice, this circumstance usually does not introduce a difficulty: most xc approximations are defined by specifying $e_{\mathrm{xc}}[n](\mathbf{r})$, and then obtaining $E_{\mathrm{xc}}[n]$ (by integration), and not the other way around. 
[202] $\rho_{2}^{(\lambda)}\left(\mathbf{r}, \mathbf{r}^{\prime}\right)$ is defined similarly to the definition of $\rho_{2}\left(\mathbf{r}, \mathbf{r}^{\prime}\right)$ in Eq. (5), where we substitute $\Psi$ with $\Psi^{(\lambda)}$.

[203] To obtain higher orders in the power expansion of $e_{\mathrm{xc}}[n](\mathbf{r})$ in $1 /|\mathbf{r}|$, one needs to employ the expansion of $\left|\mathbf{r}-\mathbf{r}^{\prime}\right|^{-1}$ in powers of $\mathbf{r}^{\prime}$ and $\mathbf{r}$ (see, e.g. [196, p. 62]) and be ready to evaluate integrals of the form $\int d^{3} r^{\prime}\left(r^{\prime}\right)^{a} \cos ^{b}\left(\widehat{\mathbf{r}, \mathbf{r}^{\prime}}\right) n_{\mathrm{xc}}\left(\mathbf{r}^{\prime} \mid \mathbf{r}\right)$.

[204] For completeness, let us mention that, in the language of exact factorization, we choose here the gauge to be $\mathbf{A}=0$, where $\mathbf{A}=\operatorname{Im}\left[\left\langle\phi \mid \nabla_{N} \phi\right\rangle\right]$.

[205] The decay rate of the KS orbitals is given, to leading order, by $\varphi_{i}(\mathbf{r}) \sim e^{-\sqrt{-2 \varepsilon_{i}}|\mathbf{r}|}$.

[206] In this last step of the derivation one has to either: (i) use the IP theorem of DFT, namely that $\varepsilon_{\text {ho }}=-I$ for the exact $\mathrm{KS}$ potential, which can be proved independently, relying on e.g. the ensemble ap- proach $[45,73,74]$; or (ii) use the fact that asymptotically the KS potential tends to zero, and not to a constant. This can be proved following Ref. [48] and [109]. In the latter case one proves the DFT IP theorem on the fly.

[207] Jacob's ladder scheme in DFT is named after the ladder Patriarch Jacob saw in his dream (Genesis 28:12-15)

[208] In this work all DFAs, including orbital-dependent ones, are considered as if they are addressed strictly within the KS approach, which results in one multiplicative KS potential, whose asymptotic behavior we examine. In orbital-dependent cases, this one potential is obtained using the OEP scheme (possibly within one of its approximations, e.g., KLI). This is although the generalized KS (GKS) approach is very common in the context of hybrids. 